\title{
Coupling of Two Partial Differential Equations and its Application
}

By

\author{
Hidetoshi TAHARA*
}

\begin{abstract}
The paper considers the following two partial differential equations
(A) $\frac{\partial u}{\partial t}=F\left(t, x, u, \frac{\partial u}{\partial x}\right)$ and
(B) $\frac{\partial w}{\partial t}=G\left(t, x, w, \frac{\partial w}{\partial x}\right)$

in the complex domain, and gives an answer to the following question: when can we say that the two equations $(\mathrm{A})$ and $(\mathrm{B})$ are equivalent? or when can we transform $(\mathrm{A})$ into (B) (or (B) into (A))? The discussion is done by considering the coupling of two equations (A) and (B), and by solving their coupling equation. The most important fact is that the coupling equation has infinitely many variables and so the meaning of the solution is not so trivial. The result is applied to the problem of analytic continuation of the solution.
\end{abstract}

\section{$\S 1 . \quad$ Introduction}

In this paper, I will present a new approach to the study of nonlinear partial differential equations in the complex domain. Since the research is still in the first stage, as a model study I will discuss only the following two partial differential equations

$$
\frac{\partial u}{\partial t}=F\left(t, x, u, \frac{\partial u}{\partial x}\right)
$$

(where $(t, x) \in \mathbb{C}^{2}$ are variables and $u=u(t, x)$ is the unknown function) and

Communicated by T. Kawai. Received May 29, 2006.

2000 Mathematics Subject Classification(s): Primary 35A22; Secondary 35A10, 35A20.

Key words: Coupling equation, equivalence of two PDEs, analytic continuation.

This research was partially supported by the Grant-in-Aid for Scientific Research No.16540169 of Japan Society for the Promotion of Science.

*Department of Mathematics, Sophia University, Kioicho, Chiyoda-ku, Tokyo 102-8554, Japan.

e-mail: h-tahara@hoffman.cc.sophia.ac.jp

(c) 2007 Research Institute for Mathematical Sciences, Kyoto University. All rights reserved. 


$$
\frac{\partial w}{\partial t}=G\left(t, x, w, \frac{\partial w}{\partial x}\right)
$$

(where $(t, x) \in \mathbb{C}^{2}$ are variables and $w=w(t, x)$ is the unknown function). For simplicity we suppose that $F\left(t, x, u_{0}, u_{1}\right)$ (resp. $\left.G\left(t, x, w_{0}, w_{1}\right)\right)$ is a holomorphic function defined in a neighborhood of the origin of $\mathbb{C}_{t} \times \mathbb{C}_{x} \times \mathbb{C}_{u_{0}} \times \mathbb{C}_{u_{1}}$ (resp. $\mathbb{C}_{t} \times \mathbb{C}_{x} \times \mathbb{C}_{w_{0}} \times \mathbb{C}_{w_{1}}$ ).

My basic question is

Question. When can we say that the two equations (A) and (B) are equivalent? or when can we transform (A) into (B) (or (B) into (A))?

One way to treat this question is to consider the coupling of (A) and (B), and to solve their coupling equation.

The coupling of two partial differential equations (A) and (B) means that we consider the following partial differential equation with infinitely many variables $\left(t, x, u_{0}, u_{1}, \ldots\right)$

$$
\frac{\partial \phi}{\partial t}+\sum_{m \geq 0} D^{m}[F]\left(t, x, u_{0}, \ldots, u_{m+1}\right) \frac{\partial \phi}{\partial u_{m}}=G(t, x, \phi, D[\phi])
$$

(where $\phi=\phi\left(t, x, u_{0}, u_{1}, \ldots\right)$ is the unknown function), or the following partial differential equation with infinitely many variables $\left(t, x, w_{0}, w_{1}, \ldots\right)$

$$
\frac{\partial \psi}{\partial t}+\sum_{m \geq 0} D^{m}[G]\left(t, x, w_{0}, \ldots, w_{m+1}\right) \frac{\partial \psi}{\partial w_{m}}=F(t, x, \psi, D[\psi])
$$

(where $\psi=\psi\left(t, x, w_{0}, w_{1}, \ldots\right)$ is the unknown function). In the equation $(\Phi)$ (resp. $(\Psi)$ ), the notation $D$ means the following vector field with infinite many variables

$$
D=\frac{\partial}{\partial x}+\sum_{i \geq 0} u_{i+1} \frac{\partial}{\partial u_{i}} \quad\left(\text { resp. } D=\frac{\partial}{\partial x}+\sum_{i \geq 0} w_{i+1} \frac{\partial}{\partial w_{i}}\right),
$$

and $D^{m}[F](m=0,1,2, \ldots)$ are defined by $D^{0}[F]=F, D[F]=D F, D^{2}[F]=$ $D(D[F]), D^{3}[F]=D\left(D^{2}[F]\right)$ and so on. These two equations $(\Phi)$ and $(\Psi)$ are called the coupling equations of (A) and (B).

In Sections 3 and 4, I will explain the meaning of the coupling of (A) and (B), solve their coupling equations, and establish the equivalence of (A) and (B) under suitable conditions. Way of solving $(\Phi)$ and $(\Psi)$ is as follows: first we 
get a formal solution and then we prove the convergence of the formal solution. Since the solution has infinitely many variables, the meaning of the convergence is not so trivial.

In the last Section 5, I will give an application to the problem of analytic continuation of the solution. The result is just the same as in Kobayashi [4] and Lope-Tahara [5]: this shows the effectiveness of our new approach in this paper.

In the case of ordinary differential equations, the theory of the coupling of two differential equations was discussed in Section 4.1 of Gérard-Tahara [1]; it will be surveyed in the next Section 2 .

The results in this paper were already announced in Tahara [9].

\section{$\S 2$. Coupling of Two Ordinary Differential Equations}

Before the discussion in partial differential equations, let us give a brief survey on the coupling of two ordinary differential equations in [Section 4.1 of Gérard-Tahara [1]].

First, let us consider the following two ordinary differential equations:

$$
\begin{aligned}
\frac{d u}{d t} & =f(t, u), \\
\frac{d w}{d t} & =g(t, w),
\end{aligned}
$$

where $f(t, u)$ (resp. $g(t, w)$ ) is a holomorphic function defined in a neighborhood of the origin of $\mathbb{C}_{t} \times \mathbb{C}_{u}$ (resp. $\left.\mathbb{C}_{t} \times \mathbb{C}_{w}\right)$.

Definition 2.1. The coupling of (a) and (b) means that we consider the following partial differential equation (2.1) or (2.2):

$$
\frac{\partial \phi}{\partial t}+f(t, u) \frac{\partial \phi}{\partial u}=g(t, \phi)
$$

(where $(t, u)$ are variables and $\phi=\phi(t, u)$ is the unknown function), or

$$
\frac{\partial \psi}{\partial t}+g(t, w) \frac{\partial \psi}{\partial w}=f(t, \psi)
$$

(where $(t, w)$ are variables and $\psi=\psi(t, w)$ is the unknown function). We call (2.1) or (2.2) the coupling equation of (a) and (b).

The convenience of considering the coupling equation lies in the following proposition. 
Proposition 2.2. (1) Let $\phi(t, u)$ be a solution of (2.1). If $u(t)$ is a solution of (a) then $w(t)=\phi(t, u(t))$ is a solution of $(\mathrm{b})$.

(2) Let $\psi(t, w)$ be a solution of (2.2). If $w(t)$ is a solution of (b) then $u(t)=\psi(t, w(t))$ is a solution of $(\mathrm{a})$.

Proof. We will prove only (1). Let $\phi(t, u)$ be a solution of $(2.1)$ and let $u(t)$ be a solution of $(\mathrm{a})$. Set $w(t)=\phi(t, u(t))$. Then we have

$$
\begin{aligned}
& \frac{d w(t)}{d t}=\frac{d}{d t} \phi(t, u(t))=\frac{\partial \phi}{\partial t}(t, u(t))+\frac{\partial \phi}{\partial u}(t, u(t)) \frac{d u(t)}{d t} \\
& =\frac{\partial \phi}{\partial t}(t, u(t))+f(t, u(t)) \frac{\partial \phi}{\partial u}(t, u(t))=g(t, \phi(t, u(t)))=g(t, w(t)) .
\end{aligned}
$$

This shows that $w(t)$ is a solution of (b).

Next, let us give a relation between two coupling equations (2.1) and (2.2). We have

Proposition 2.3. $\quad$ (1) Let $\phi(t, u)$ be a solution of (2.1) and suppose that the relation $w=\phi(t, u)$ is equivalent to $u=\psi(t, w)$ for some function $\psi(t, w)$; then $\psi(t, w)$ is a solution of $(2.2)$.

(2) Let $\psi(t, w)$ be a solution of (2.2) and suppose that the relation $u=$ $\psi(t, w)$ is equivalent to $w=\phi(t, u)$ for some function $\phi(t, u)$; then $\phi(t, u)$ is a solution of (2.1).

Proof. We will show only the part (1). Since $w=\phi(t, u)$ is equivalent to $u=\psi(t, w)$, we get $u \equiv \psi(t, \phi(t, u))$. By derivating this with respect to $t$ and $u$ we get

$$
\begin{aligned}
0 & \equiv \frac{\partial \psi}{\partial t}(t, \phi(t, u))+\frac{\partial \psi}{\partial w}(t, \phi(t, u)) \frac{\partial \phi}{\partial t}(t, u), \\
1 & \equiv \frac{\partial \psi}{\partial w}(t, \phi(t, u)) \frac{\partial \phi}{\partial u}(t, u) .
\end{aligned}
$$

By using these relations we have

$$
\begin{aligned}
\left(\frac{\partial \psi}{\partial t}\right. & \left.+g(t, w) \frac{\partial \psi}{\partial w}\right)\left.\right|_{w=\phi(t, u)}=\frac{\partial \psi}{\partial t}(t, \phi(t, u))+g(t, \phi(t, u)) \frac{\partial \psi}{\partial w}(t, \phi(t, u)) \\
& =-\frac{\partial \psi}{\partial w}(t, \phi(t, u)) \frac{\partial \phi}{\partial t}(t, u)+g(t, \phi(t, u)) \frac{\partial \psi}{\partial w}(t, \phi(t, u)) \\
& =\frac{\partial \psi}{\partial w}(t, \phi(t, u))\left(-\frac{\partial \phi}{\partial t}(t, u)+g(t, \phi(t, u))\right) \\
& =\frac{\partial \psi}{\partial w}(t, \phi(t, u)) f(t, u) \frac{\partial \phi}{\partial u}(t, u)=f(t, u) .
\end{aligned}
$$


Since $w=\phi(t, u)$ is equivalent to $u=\psi(t, w)$, we obtain

$$
\frac{\partial \psi}{\partial t}+g(t, w) \frac{\partial \psi}{\partial w}=f(t, \psi(t, w))
$$

this proves the result (1).

Now, let us discuss the equivalence of two differential equations. Let $f(t, z)$ and $g(t, z)$ be holomorphic functions of $(t, z)$ in a neighborhood of $(0,0) \in$ $\mathbb{C}_{t} \times \mathbb{C}_{z}$ as before, and let us consider the following two equations:

$$
\begin{aligned}
& \text { [a] } \frac{d u}{d t}=f(t, u), \quad u(t) \longrightarrow 0 \quad(\text { as } t \longrightarrow 0) \text {, } \\
& \text { [b] } \quad \frac{d w}{d t}=g(t, w), \quad w(t) \longrightarrow 0 \quad(\text { as } t \longrightarrow 0) .
\end{aligned}
$$

Denote by $\mathcal{S}_{a}$ (resp. $\mathcal{S}_{b}$ ) the set of all the holomorphic solutions of [a] (resp. [b]) in a suitable sectorial neighborhood of $t=0$. Let $\phi(t, u)$ be a holomorphic solution of (2.1) satisfying $\phi(0,0)=0$ : if $u(t) \in \mathcal{S}_{a}$ we have $\phi(t, u(t)) \longrightarrow$ $\phi(0,0)=0($ as $t \longrightarrow 0)$, and therefore the mapping

$$
\Phi: \mathcal{S}_{a} \ni u(t) \longrightarrow \phi(t, u(t)) \in \mathcal{S}_{b}
$$

is well defined. Hence, if $\Phi: \mathcal{S}_{a} \longrightarrow \mathcal{S}_{b}$ is bijective, solving [a] is equivalent to solving [b]. Thus:

Definition 2.4. If $\Phi: \mathcal{S}_{a} \longrightarrow \mathcal{S}_{b}$ is well defined and bijective, we say that the two equations [a] and [b] are equivalent.

The following result gives a sufficient condition for $\Phi$ to be bijective.

Theorem 2.5. If the coupling equation (2.1) has a holomorphic solution $\phi(t, u)$ in a neighborhood of $(0,0) \in \mathbb{C}_{t} \times \mathbb{C}_{u}$ satisfying $\phi(0,0)=0$ and $(\partial \phi / \partial u)(0,0) \neq 0$, then the mapping $\Phi$ is bijective and so the two equations [a] and $[\mathrm{b}]$ are equivalent.

Proof. Let us show that the mapping $\Phi: \mathcal{S}_{a} \longrightarrow \mathcal{S}_{b}$ is bijective. Since $(\partial \phi / \partial u)(0,0) \neq 0$, by the implicit function theorem we can solve the equation $w=\phi(t, u)$ with respect to $u$ and obtain $u=\psi(t, w)$ for some holomorphic function $\psi(t, w)$ satisfying $\psi(0,0)=0$. Moreover we know that this $\psi(t, w)$ is a solution of (2.2). Therefore, by (2) of Proposition 2.2 we can define the mapping

$$
\Psi: \mathcal{S}_{b} \ni w(t) \longrightarrow \psi(t, w(t)) \in \mathcal{S}_{a}
$$


Since $w=\phi(t, u)$ is equivalent to $u=\psi(t, w), w(t)=\phi(t, u(t))$ is also equivalent to $u(t)=\psi(t, w(t))$; this implies that $w(t)=\Phi(u(t))$ is equivalent to $u(t)=$ $\Psi(w(t))$. Thus we can conclude that $\Phi: \mathcal{S}_{a} \longrightarrow \mathcal{S}_{b}$ is bijective and $\Psi$ is the inverse mapping of $\Phi$.

In the application, we will regard (2.3) as an original equation and (2.4) as a reduced equation. Then, what we must do is to find a good reduced form (2.4) so that the following 1) and 2) are satisfied:

1) (2.3) is equivalent to $(2.4)$, and

$2)$ we can solve (2.4) directly.

In a sense, (2.4) must be the best partner, and our coupling equation is a tool to find its best partner. The naming "coupling" comes from such a meaning.

In Gérard-Tahara [1], one can see many concrete applications of this theory to the reduction problem of Briot-Bouquet's differential equation.

\section{$\S 3 . \quad$ Coupling of Two Partial Differential Equations}

Now, let us generalize the theory in Section 2 to partial differential equations. In this section we will give only a formal theory, and in the next section we will give a substantial meaning to the formal theory.

Let us consider the following two nonlinear partial differential equations:

$$
\frac{\partial u}{\partial t}=F\left(t, x, u, \frac{\partial u}{\partial x}\right)
$$

(where $(t, x) \in \mathbb{C}^{2}$ are variables and $u=u(t, x)$ is the unknown function) and

$$
\frac{\partial w}{\partial t}=G\left(t, x, w, \frac{\partial w}{\partial x}\right)
$$

(where $(t, x) \in \mathbb{C}^{2}$ are variables and $w=w(t, x)$ is the unknown function). For simplicity we suppose that $F\left(t, x, u_{0}, u_{1}\right)$ (resp. $\left.G\left(t, x, w_{0}, w_{1}\right)\right)$ is a holomorphic function defined in a neighborhood of the origin of $\mathbb{C}_{t} \times \mathbb{C}_{x} \times \mathbb{C}_{u_{0}} \times \mathbb{C}_{u_{1}}$ (resp. $\mathbb{C}_{t} \times \mathbb{C}_{x} \times \mathbb{C}_{w_{0}} \times \mathbb{C}_{w_{1}}$ ).

For a function $\phi=\phi\left(t, x, u_{0}, u_{1}, \ldots\right)$ with respect to the infinitely many variables $\left(t, x, u_{0}, u_{1}, \ldots\right)$ we define $D[\phi]\left(t, x, u_{0}, u_{1}, \ldots\right)$ by

$$
D[\phi]=\frac{\partial \phi}{\partial x}\left(t, x, u_{0}, u_{1}, \ldots\right)+\sum_{i \geq 0} u_{i+1} \frac{\partial \phi}{\partial u_{i}}\left(t, x, u_{0}, u_{1}, \ldots\right) .
$$

For $m \geq 2$ we define $D^{m}[\phi]$ as follows: $D^{2}[\phi]=D[D[\phi]], D^{3}[\phi]=D\left[D^{2}[\phi]\right]$, and so on. If $\phi$ is a function with $(p+3)$-variables $\left(t, x, u_{0}, \ldots, u_{p}\right)$ then $D^{m}[\phi]$ 
is a function with $(p+m+3)$-variables $\left(t, x, u_{0}, \ldots, u_{p+m}\right)$. Of course, if $\psi=$ $\psi\left(t, x, w_{0}, w_{1}, \ldots\right)$ is a function with respect to the variables $\left(t, x, w_{0}, w_{1}, \ldots\right)$ the notation $D[\psi]\left(t, x, w_{0}, w_{1}, \ldots\right)$ is read as

$$
D[\psi]=\frac{\partial \psi}{\partial x}\left(t, x, w_{0}, w_{1}, \ldots\right)+\sum_{i \geq 0} w_{i+1} \frac{\partial \psi}{\partial w_{i}}\left(t, x, w_{0}, w_{1}, \ldots\right) .
$$

We can regard $D$ as a vector field with infinitely many variables $\left(x, u_{0}, u_{1}, \ldots\right)$ (resp. $\left.\left(x, w_{0}, w_{1}, \ldots\right)\right)$ :

$$
D=\frac{\partial}{\partial x}+\sum_{i \geq 0} u_{i+1} \frac{\partial}{\partial u_{i}} \quad\left(\text { resp. } D=\frac{\partial}{\partial x}+\sum_{i \geq 0} w_{i+1} \frac{\partial}{\partial w_{i}}\right)
$$

This operator $D$ comes from the following formula: if $K\left(t, x, u_{0}, u_{1}, \ldots\right)$ is a function with infinitely many variables $\left(t, x, u_{0}, u_{1}, \ldots\right)$ and if $u(x)$ is a holomorphic function, then under the relation $u_{i}=(\partial / \partial x)^{i} u(i=0,1,2, \ldots)$ we have

$$
\frac{\partial}{\partial x} K\left(t, x, u, \frac{\partial u}{\partial x}, \ldots\right)=\frac{\partial K}{\partial x}+\frac{\partial K}{\partial u_{0}} u_{1}+\frac{\partial K}{\partial u_{1}} u_{2}+\cdots=D[K] .
$$

Therefore, for any $m \in \mathbb{N}$ we have

$$
D^{m}[K]\left(t, x, u, \frac{\partial u}{\partial x}, \ldots\right)=\left(\frac{\partial}{\partial x}\right)^{m}\left[K\left(t, x, u, \frac{\partial u}{\partial x}, \ldots\right)\right] .
$$

Definition 3.0.1. The coupling of two partial differential equations (A) and (B) means that we consider the following partial differential equation with infinitely many variables $\left(t, x, u_{0}, u_{1}, \ldots\right)$

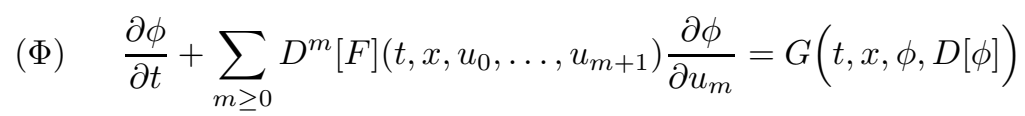

(where $\phi=\phi\left(t, x, u_{0}, u_{1}, \ldots\right)$ is the unknown function), or the following partial differential equation with infinitely many variables $\left(t, x, w_{0}, w_{1}, \ldots\right)$

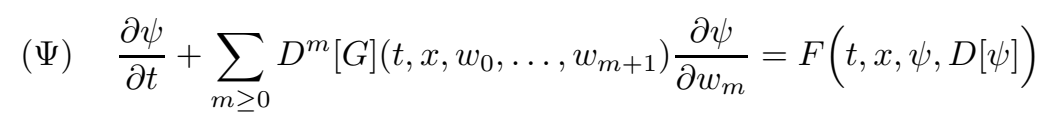

(where $\psi=\psi\left(t, x, w_{0}, w_{1}, \ldots\right)$ is the unknown function). We call $(\Phi)$ or $(\Psi)$ the coupling equation of $(\mathrm{A})$ and $(\mathrm{B})$. 


\section{§3.1. The formal meaning of the coupling equation}

In this section we will explain the meaning of the coupling equations $(\Phi)$ and $(\Psi)$ in the formal sense. Here, "in the formal sense" means that the result is true if the formal calculation makes sense. For simplicity we write

$$
\phi(t, x, u, \partial u / \partial x, \ldots)=\phi\left(t, x, u, \frac{\partial u}{\partial x}, \frac{\partial^{2} u}{\partial x^{2}}, \ldots, \frac{\partial^{n} u}{\partial x^{n}}, \ldots\right) .
$$

The convenience of considering the coupling equation lies in the following proposition.

Proposition 3.1.1. (1) If $\phi\left(t, x, u_{0}, u_{1}, \ldots\right)$ is a solution of $(\Phi)$ and if $u(t, x)$ is a solution of $(\mathrm{A})$, then the function $w(t, x)=\phi(t, x, u, \partial u / \partial x, \ldots)$ is a solution of $(\mathrm{B})$.

(2) If $\psi\left(t, x, w_{0}, w_{1}, \ldots\right)$ is a solution of $(\Psi)$ and if $w(t, x)$ is a solution of (B), then the function $u(t, x)=\psi(t, x, w, \partial w / \partial x, \ldots)$ is a solution of $(\mathrm{A})$.

Proof. We will show only (1). Let $\phi\left(t, x, u_{0}, u_{1}, \ldots\right)$ be a solution $(\Phi)$ and let $u(t, x)$ be a solution of $(\mathrm{A})$. Set $u_{i}(t, x)=(\partial / \partial x)^{i} u(t, x)(i=0,1,2, \ldots)$ : we have $w(t, x)=\phi(t, x, u, \partial u / \partial x, \ldots)=\phi\left(t, x, u_{0}, u_{1}, \ldots\right)$ and $\partial w / \partial x=D[\phi](t, x$, $\left.u_{0}, u_{1}, \ldots\right)$. Therefore we have

$$
\begin{aligned}
\frac{\partial w}{\partial t} & =\frac{\partial \phi}{\partial t}+\sum_{i \geq 0} \frac{\partial \phi}{\partial u_{i}} \frac{\partial u_{i}}{\partial t}=\frac{\partial \phi}{\partial t}+\sum_{i \geq 0} \frac{\partial \phi}{\partial u_{i}}\left(\frac{\partial}{\partial x}\right)^{i}\left[\frac{\partial u}{\partial t}\right] \\
& =\frac{\partial \phi}{\partial t}+\sum_{i \geq 0} \frac{\partial \phi}{\partial u_{i}}\left(\frac{\partial}{\partial x}\right)^{i}\left[F\left(t, x, u, \frac{\partial u}{\partial x}\right)\right] \\
& =\frac{\partial \phi}{\partial t}+\sum_{i \geq 0} \frac{\partial \phi}{\partial u_{i}} D^{i}[F]\left(t, x, u_{0}, \ldots, u_{i+1}\right) \\
& =G(t, x, \phi, D[\phi])=G\left(t, x, w, \frac{\partial w}{\partial x}\right) .
\end{aligned}
$$

This shows that $w(t, x)$ is a solution of $(\mathrm{B})$.

In order to state the relation between $(\Phi)$ and $(\Psi)$, let us introduce the notion of the reversibility of $\phi\left(t, x, u_{0}, u_{1}, \ldots\right)$.

Definition 3.1.2. Let $\phi\left(t, x, u_{0}, u_{1}, \ldots\right)$ be a function in $\left(t, x, u_{0}, u_{1}, \ldots\right)$. We say that the relation $w=\phi(t, x, u, \partial u / \partial x, \ldots)$ is reversible with respect to $u$ and $w$ if there is a function $\psi\left(t, x, w_{0}, w_{1}, \ldots\right)$ in $\left(t, x, w_{0}, w_{1}, \ldots\right)$ such that 
the relation

$$
\left\{\begin{aligned}
w_{0}= & \phi\left(t, x, u_{0}, u_{1}, u_{2}, \ldots\right) \\
w_{1}= & D[\phi]\left(t, x, u_{0}, u_{1}, u_{2}, \ldots\right) \\
w_{2}= & D^{2}[\phi]\left(t, x, u_{0}, u_{1}, u_{2}, \ldots\right) \\
& \ldots \ldots \\
& \ldots \ldots
\end{aligned}\right.
$$

is equivalent to

$$
\left\{\begin{aligned}
u_{0}= & \psi\left(t, x, w_{0}, w_{1}, w_{2}, \ldots\right) \\
u_{1}= & D[\psi]\left(t, x, w_{0}, w_{1}, w_{2}, \ldots\right) \\
u_{2}= & D^{2}[\psi]\left(t, x, w_{0}, w_{1}, w_{2}, \ldots\right) \\
& \ldots \ldots \\
& \ldots \ldots
\end{aligned}\right.
$$

In this case the function $\psi\left(t, x, w_{0}, w_{1}, \ldots\right)$ is called the reverse function of $\phi\left(t, x, u_{0}, u_{1}, \ldots\right)$.

By the definition, we see

Lemma 3.1.3. The reverse function $\psi\left(t, x, w_{0}, w_{1}, \ldots\right)$ of $\phi\left(t, x, u_{0}, u_{1}\right.$, ...) is unique, if it exists.

Proof. Let $\psi_{1}\left(t, x, w_{0}, w_{1}, \ldots\right)$ be another reverse function of $\phi\left(t, x, u_{0}, u_{1}\right.$, ...). Then the system

$$
\left\{\begin{aligned}
u_{0}= & \psi_{1}\left(t, x, w_{0}, w_{1}, w_{2}, \ldots\right) \\
u_{1}= & D\left[\psi_{1}\right]\left(t, x, w_{0}, w_{1}, w_{2}, \ldots\right) \\
u_{2}= & D^{2}\left[\psi_{1}\right]\left(t, x, w_{0}, w_{1}, w_{2}, \ldots\right) \\
& \ldots \ldots \\
& \ldots \ldots
\end{aligned}\right.
$$

is equivalent to (3.1.1), and so (3.1.2) and (3.1.3) are equivalent; this means that the equality

$$
\psi\left(t, x, w_{0}, w_{1}, w_{2}, \ldots\right)=\psi_{1}\left(t, x, w_{0}, w_{1}, w_{2}, \ldots\right)
$$

holds as functions with respect to $\left(t, x, w_{0}, w_{1}, w_{2}, \ldots\right)$.

The following proposition gives the relation between two coupling equations $(\Phi)$ and $(\Psi)$ : we can say that the equation $(\Psi)$ is the reverse of $(\Phi)$. 
Proposition 3.1.4. If $\phi\left(t, x, u_{0}, u_{1}, \ldots\right)$ is a solution of $(\Phi)$ and if the relation $w=\phi(t, x, u, \partial u / \partial x, \ldots)$ is reversible with respect to $u$ and $w$, then the reverse function $\psi\left(t, x, w_{0}, w_{1}, \ldots\right)$ is a solution of $(\Psi)$.

To prove this we need the following lemma:

Lemma 3.1.5. (1) For any functions $\phi\left(t, x, u_{0}, u_{1}, \ldots\right)$ and $H\left(t, x, u_{0}\right.$, $\left.u_{1}, \ldots\right)$ we have

$$
\sum_{i \geq 0} D^{k}\left[\frac{\partial \phi}{\partial u_{i}} D^{i}[H]\right]=\sum_{i \geq 0} \frac{\partial D^{k}[\phi]}{\partial u_{i}} D^{i}[H], \quad k=0,1,2, \ldots
$$

(2) For a function $K\left(t, x, w_{0}, w_{1}, \ldots\right)$ with respect to the variables $(t, x$, $\left.w_{0}, w_{1}, \ldots\right)$ we write $K_{\phi}=K(t, x, \phi, D[\phi], \ldots)$ which is a function with respect to the variables $\left(t, x, u_{0}, u_{1}, \ldots\right)$. We have

$$
D^{k}\left[K_{\phi}\right]=\left(D_{w}{ }^{k}[K]\right)_{\phi}, \quad k=0,1,2, \ldots,
$$

where $D_{w}$ is the same operator as $D$ with $\left(u_{0}, u_{1}, \ldots\right)$ replaced by $\left(w_{0}, w_{1}, \ldots\right)$.

(3) If $\phi\left(t, x, u_{0}, u_{1}, \ldots\right)$ is a solution of the coupling equation $(\Phi)$, we have

$$
\frac{\partial D^{k}[\phi]}{\partial t}+\sum_{i \geq 0} D^{i}[F] \frac{\partial D^{k}[\phi]}{\partial u_{i}}=\left(D_{w}{ }^{k}[G]\right)_{\phi}, \quad k=0,1,2, \ldots
$$

as a function with respect to the variable $\left(t, x, u_{0}, u_{1}, \ldots\right)$.

Proof. Let us show (1). By the definition of the operator $D$ we have

$$
\begin{aligned}
\frac{\partial}{\partial u_{i}} D[\phi] & =\frac{\partial}{\partial u_{i}}\left(\frac{\partial \phi}{\partial x}+u_{1} \frac{\partial \phi}{\partial u_{0}}+\cdots+u_{i} \frac{\partial \phi}{\partial u_{i-1}}+\cdots\right) \\
& =D\left[\frac{\partial \phi}{\partial u_{i}}\right]+\frac{\partial \phi}{\partial u_{i-1}} \quad \text { for } i=0,1,2, \ldots
\end{aligned}
$$

(where $\partial \phi / \partial u_{-1}=0$ ); therefore we obtain

$$
\begin{aligned}
& \sum_{i \geq 0} D\left[\frac{\partial \phi}{\partial u_{i}} D^{i}[H]\right]=\sum_{i \geq 0}\left(D\left[\frac{\partial \phi}{\partial u_{i}}\right] D^{i}[H]+\frac{\partial \phi}{\partial u_{i}} D^{i+1}[H]\right) \\
& =\sum_{i \geq 0}\left(\left(\frac{\partial D[\phi]}{\partial u_{i}}-\frac{\partial \phi}{\partial u_{i-1}}\right) D^{i}[H]+\frac{\partial \phi}{\partial u_{i}} D^{i+1}[H]\right) \\
& =\sum_{i \geq 0} \frac{\partial D[\phi]}{\partial u_{i}} D^{i}[H]-\sum_{i \geq 1} \frac{\partial \phi}{\partial u_{i-1}} D^{i}[H]+\sum_{i \geq 0} \frac{\partial \phi}{\partial u_{i}} D^{i+1}[H] \\
& =\sum_{i \geq 0} \frac{\partial D[\phi]}{\partial u_{i}} D^{i}[H] .
\end{aligned}
$$


This proves the equality (3.1.4) in the case $k=1$. The general case $k \geq 2$ can be proved by induction on $k$.

Next let us show (2). The case $k=1$ is verified by

$$
\begin{aligned}
D\left[K_{\phi}\right] & =\frac{\partial K_{\phi}}{\partial x}+\sum_{i \geq 0} u_{i+1} \frac{\partial K_{\phi}}{\partial u_{i}} \\
& =\left(\frac{\partial K}{\partial x}\right)_{\phi}+\sum_{j \geq 0}\left(\frac{\partial K}{\partial w_{j}}\right)_{\phi} \frac{\partial D^{j}[\phi]}{\partial x}+\sum_{i \geq 0} u_{i+1} \sum_{j \geq 0}\left(\frac{\partial K}{\partial w_{j}}\right)_{\phi} \frac{\partial D^{j}[\phi]}{\partial u_{i}} \\
& =\left(\frac{\partial K}{\partial x}\right)_{\phi}+\sum_{j \geq 0}\left(\frac{\partial K}{\partial w_{j}}\right)_{\phi}\left[\frac{\partial D^{j}[\phi]}{\partial x}+\sum_{i \geq 0} u_{i+1} \frac{\partial D^{j}[\phi]}{\partial u_{i}}\right] \\
& =\left(\frac{\partial K}{\partial x}\right)_{\phi}+\sum_{j \geq 0}\left(\frac{\partial K}{\partial w_{j}}\right)_{\phi} D^{j+1}[\phi] \\
& =\left(\frac{\partial K}{\partial x}+\sum_{j \geq 0} \frac{\partial K}{\partial w_{j}} w_{j+1}\right)_{\phi}=\left(D_{w}[K]\right)_{\phi} .
\end{aligned}
$$

The general case $k \geq 2$ can be proved by induction on $k$.

Lastly let us show (3). Since $\phi\left(t, x, u_{0}, u_{1}, \ldots\right)$ satisfies the equation $(\Phi)$, by applying $D^{k}$ to $(\Phi)$ and by using the relation $D^{k}\left[G_{\phi}\right]=\left(D_{w}{ }^{k}[G]\right)_{\phi}$ we have

$$
\frac{\partial D^{k}[\phi]}{\partial t}+\sum_{i \geq 0} D^{k}\left[D^{i}[F] \frac{\partial \phi}{\partial u_{i}}\right]=\left(D_{w}{ }^{k}[G]\right)_{\phi} .
$$

Therefore, to prove the equality (3.1.6) we have only to notice the following equality:

$$
\sum_{i \geq 0} D^{k}\left[D^{i}[F] \frac{\partial \phi}{\partial u_{i}}\right]=\sum_{i \geq 0} D^{i}[F] \frac{\partial D^{k}[\phi]}{\partial u_{i}}, \quad k=0,1,2, \ldots
$$

which is already proved in the part (1).

Proof of Proposition 3.1.4. Since (3.1.1) and (3.1.2) are equivalent, we have the equality

$$
u_{0}=\psi\left(t, x, \phi, D[\phi], D^{2}[\phi], \ldots\right)
$$

as a function with respect to the variables $\left(t, x, u_{0}, u_{1}, u_{2}, \ldots\right)$. Therefore, by applying $\partial / \partial u_{i}$ to (3.1.7) we have

$$
\sum_{j \geq 0}\left(\frac{\partial \psi}{\partial w_{j}}\right)_{\phi} \frac{\partial D^{j}[\phi]}{\partial u_{i}}= \begin{cases}1, & \text { if } i=0 \\ 0, & \text { if } i \geq 1\end{cases}
$$


Also, by applying $\partial / \partial t$ to (3.1.7) and then by using (3.1.6) and (3.1.8) we have

$$
\begin{aligned}
0 & =\left(\frac{\partial \psi}{\partial t}\right)_{\phi}+\sum_{j \geq 0}\left(\frac{\partial \psi}{\partial w_{j}}\right)_{\phi} \frac{\partial D^{j}[\phi]}{\partial t} \\
& =\left(\frac{\partial \psi}{\partial t}\right)_{\phi}+\sum_{j \geq 0}\left(\frac{\partial \psi}{\partial w_{j}}\right)_{\phi}\left[-\sum_{i \geq 0} D^{i}[F] \frac{\partial D^{j}[\phi]}{\partial u_{i}}+\left(D_{w}{ }^{j}[G]\right)_{\phi}\right] \\
& =\left(\frac{\partial \psi}{\partial t}\right)_{\phi}+\sum_{j \geq 0}\left(\frac{\partial \psi}{\partial w_{j}}\right)_{\phi}\left(D_{w}{ }^{j}[G]\right)_{\phi}-\sum_{i \geq 0} D^{i}[F] \sum_{j \geq 0}\left(\frac{\partial \psi}{\partial w_{j}}\right)_{\phi} \frac{\partial D^{j}[\phi]}{\partial u_{i}} \\
& =\left(\frac{\partial \psi}{\partial t}\right)_{\phi}+\sum_{j \geq 0}\left(\frac{\partial \psi}{\partial w_{j}}\right)_{\phi}\left(D_{w}{ }^{j}[G]\right)_{\phi}-F
\end{aligned}
$$

Hence, as a function with respect to the variables $\left(t, x, u_{0}, u_{1}, u_{1}, \ldots\right)$ we have the equality

$$
\left(\frac{\partial \psi}{\partial t}\right)_{\phi}+\sum_{j \geq 0}\left(\frac{\partial \psi}{\partial w_{j}}\right)_{\phi}\left(D_{w}^{j}[G]\right)_{\phi}=F\left(t, x, u_{0}, u_{1}\right) .
$$

Since (3.1.1) and (3.1.2) are equivalent, we can regard this equality as a function with respect to the variables $\left(t, x, w_{0}, w_{1}, \ldots\right)$ and obtain

$$
\frac{\partial \psi}{\partial t}+\sum_{j \geq 0} \frac{\partial \psi}{\partial w_{j}} D^{j}[G]=F(t, x, \psi, D[\psi]) .
$$

This proves that $\psi\left(t, x, w_{0}, w_{1}, \ldots\right)$ is a solution of the equation $(\Psi)$.

\section{§3.2. Equivalence of (A) and (B)}

Let $\mathscr{F}$ and $\mathscr{G}$ be function spaces in which we can consider the following two partial differential equations:

$$
\begin{aligned}
{[\mathrm{A}] } & \frac{\partial u}{\partial t} & =F\left(t, x, u, \frac{\partial u}{\partial x}\right) \text { in } \mathscr{F}, \\
\text { [B] } & \frac{\partial w}{\partial t} & =G\left(t, x, w, \frac{\partial w}{\partial x}\right) \text { in } \mathscr{G} .
\end{aligned}
$$

Set

$$
\begin{aligned}
& \mathcal{S}_{A}=\text { the set of all solutions of }[\mathrm{A}] \text { in } \mathscr{F}, \\
& \mathcal{S}_{B}=\text { the set of all solutions of }[\mathrm{B}] \text { in } \mathscr{G}
\end{aligned}
$$


Then, if the coupling equation $(\Phi)$ has a solution $\phi\left(t, x, u_{0}, u_{1} \ldots\right)$ and if $\phi(t, x$, $u, \partial u / \partial x, \ldots) \in \mathscr{G}$ is well defined for any $u \in \mathcal{S}_{A}$, we can define the mapping

$$
\Phi: \mathcal{S}_{A} \ni u(t, x) \longmapsto w(t, x)=\phi(t, x, u, \partial u / \partial x, \ldots) \in \mathcal{S}_{B} .
$$

If the relation $w=\phi(t, x, u, \partial u / \partial x, \ldots)$ is reversible with respect to $u$ and $w$, and if the reverse function $\psi\left(t, x, w_{0}, w_{1}, \ldots\right)$ satisfies $\psi(t, x, w, \partial w / \partial x, \ldots) \in \mathscr{F}$ for any $w \in \mathcal{S}_{B}$, we can also define the mapping

$$
\Psi: \mathcal{S}_{B} \ni w(t, x) \longmapsto u(t, x)=\psi(t, x, w, \partial w / \partial x, \ldots) \in \mathcal{S}_{A} .
$$

Set $w(t, x)=\phi(t, x, u, \partial u / \partial x, \ldots)$; then by the definition of $D$ we have

$$
\left\{\begin{array}{l}
w=\phi(t, x, u, \partial u / \partial x, \ldots), \\
\partial w / \partial x=D[\phi](t, x, u, \partial u / \partial x, \ldots), \\
\partial^{2} w / \partial x^{2}=D^{2}[\phi](t, x, u, \partial u / \partial x, \ldots), \\
\cdots \cdots \\
\cdots \cdots
\end{array}\right.
$$

Similarly, if we set $u(t, x)=\psi(t, x, w, \partial w / \partial x, \ldots)$ we have

$$
\left\{\begin{array}{l}
u=\phi(t, x, w, \partial w / \partial x, \ldots) \\
\partial u / \partial x=D[\phi](t, x, w, \partial w / \partial x, \ldots) \\
\partial^{2} u / \partial x^{2}=D^{2}[\phi](t, x, w, \partial w / \partial x, \ldots) \\
\ldots \ldots \\
\ldots \ldots
\end{array}\right.
$$

Since (3.1.1) is equivalent to (3.1.2) we have the equivalence between (3.2.3) and (3.2.4); therefore we have $\Psi \circ \Phi=$ identity in $\mathcal{S}_{A}$ and $\Phi \circ \Psi=$ identity in $\mathcal{S}_{B}$. Thus, we obtain

Theorem 3.2.1. Suppose that the coupling equation $(\Phi)$ has a solution $\phi\left(t, x, u_{0}, u_{1} \ldots\right)$ and that the relation $w=\phi(t, x, u, \partial u / \partial x, \ldots)$ is reversible with respect to $u$ and $w$. If both mappings (3.2.1) and (3.2.2) are well defined, we can conclude that the both mappings are bijective and that one is the inverse of the other.

By this theorem, we may say:

Definition 3.2.2. (1) If the coupling equation $(\Phi)$ (resp. $(\Psi)$ ) has a solution $\phi\left(t, x, u_{0}, u_{1} \ldots\right)$ (resp. $\left.\psi\left(t, x, w_{0}, w_{1} \ldots\right)\right)$ and if the relation $w=$ $\phi(t, x, u, \partial u / \partial x, \ldots)(\operatorname{resp} . \quad u=\psi(t, x, w, \partial w / \partial x, \ldots))$ is reversible with respect to $u$ and $w$ (or $w$ and $u$ ), then we say that the two equations (A) and (B) are formally equivalent. 
(2) In addition, if both mappings (3.2.1) and (3.2.2) are well defined, we say that the two equations $[\mathrm{A}]$ and $[\mathrm{B}]$ are equivalent.

In the application, we will regard $[\mathrm{A}]$ as an original equation and $[\mathrm{B}]$ as a reduced equation. Then, what we must do is to find a good reduced form $[\mathrm{B}]$ so that the following 1) and 2) are satisfied:

1) $[\mathrm{A}]$ is equivalent to $[\mathrm{B}]$, and

2) we can solve $[\mathrm{B}]$ concretely.

\section{§3.3. A Sufficient condition for the reversibility}

As is seen above, the condition of the reversibility of $\phi\left(t, x, u_{0}, u_{1}, \ldots\right)$ is very important. In this section we will give a sufficient condition for the relation $w=\phi(t, x, u, \partial u / \partial x, \ldots)$ to be reversible with respect to $u$ and $w$.

Let us introduce the notations: $D_{R}=\{x \in \mathbb{C} ;|x| \leq R\}, \mathcal{O}_{R}$ denotes the ring of holomorphic functions in a neighborhood of $D_{R}$, and $\mathcal{O}_{R}\left[\left[u_{0}, \ldots, u_{p}\right]\right]$ denotes the ring of formal power series in $\left(u_{0}, \ldots, u_{p}\right)$ with coefficients in $\mathcal{O}_{R}$.

Proposition 3.3.1. If $\phi\left(t, x, u_{0}, u_{1}, \ldots\right)$ is of the form

$$
\phi=u_{0}+\sum_{k \geq 1} \phi_{k}\left(x, u_{0}, \ldots, u_{k}\right) t^{k} \in \sum_{k \geq 0} \mathcal{O}_{R}\left[\left[u_{0}, \ldots, u_{k}\right]\right] t^{k},
$$

the relation $w=\phi(t, x, u, \partial u / \partial x, \ldots)$ is reversible with respect to $u$ and $w$, and the reverse function $\psi\left(t, x, w_{0}, w_{1}, \ldots\right)$ is also of the form

$$
\psi=w_{0}+\sum_{k \geq 1} \psi_{k}\left(x, w_{0}, \ldots, w_{k}\right) t^{k} \in \sum_{k \geq 0} \mathcal{O}_{R}\left[\left[w_{0}, \ldots, w_{k}\right]\right] t^{k} .
$$

To prove this, let us consider the following equation with respect to the unknown function $\psi=\psi\left(t, x, w_{0}, w_{1}, \ldots\right)$ :

$$
w_{0}=\phi\left(t, x, \psi, D[\psi], D^{2}[\psi], \ldots\right) \quad \text { in } \sum_{k \geq 0} \mathcal{O}_{R}\left[\left[w_{0}, \ldots, w_{k}\right]\right] t^{k} .
$$

We have

Lemma 3.3.2. Let $\phi\left(t, x, u_{0}, u_{1}, \ldots\right)$ be of the form (3.3.1). Then, the equation (3.3.3) has a unique solution $\psi\left(t, x, w_{0}, w_{1}, \ldots\right)$ of the form (3.3.2) and it satisfies also

$$
u_{0}=\psi\left(t, x, \phi, D[\phi], D^{2}[\phi], \ldots\right) \quad \text { in } \sum_{k \geq 0} \mathcal{O}_{R}\left[\left[u_{0}, \ldots, u_{k}\right]\right] t^{k} .
$$


Proof. Since the equation (3.3.3) is written in the form

$$
w_{0}=\psi+\sum_{k \geq 1} \phi_{k}\left(x, \psi, D[\psi], \ldots, D^{k}[\psi]\right) t^{k}
$$

under the expression $\psi=\sum_{i \geq 0} \psi_{i} t^{i}$ we have

$$
w_{0}=\sum_{i \geq 0} \psi_{i} t^{i}+\sum_{k \geq 1} \phi_{k}\left(x, \sum_{i \geq 0} \psi_{i} t^{i}, \ldots, \sum_{i \geq 0} D^{k}\left[\psi_{i}\right] t^{i}\right) t^{k} .
$$

By setting $t=0$ we have $\psi_{0}=w_{0}$, and so this equation is reduced to the form

$$
\sum_{i \geq 1} \psi_{i} t^{i}=-\sum_{k \geq 1} \phi_{k}\left(x, \sum_{i \geq 0} \psi_{i} t^{i}, \ldots, \sum_{i \geq 0} D^{k}\left[\psi_{i}\right] t^{i}\right) t^{k} .
$$

Let us look at the coefficients of $t$ in the both sides of (3.3.5); we have $\psi_{1}=-\phi_{1}\left(x, \psi_{0}, D\left[\psi_{0}\right]\right)=-\phi_{1}\left(x, w_{0}, D\left[w_{0}\right]\right)=-\phi_{1}\left(x, w_{0}, w_{1}\right)$ (in the second equality we used the fact $\left.\psi_{0}=w_{0}\right)$.

In general, for $p \geq 1$ we consider the equation (3.3.5) in the modulo class $O\left(t^{p+1}\right)$; then we have

$$
(3.3 .6)_{p} \sum_{i=1}^{p} \psi_{i} t^{i} \equiv-\sum_{k=1}^{p} \phi_{k}\left(x, \sum_{i=0}^{p-k} \psi_{i} t^{i}, \ldots, \sum_{i=0}^{p-k} D^{k}\left[\psi_{i}\right] t^{i}\right) t^{k} \bmod O\left(t^{p+1}\right) .
$$

Note that only the terms $\psi_{0}\left(=w_{0}\right), \psi_{1}, \ldots, \psi_{p-1}$ appear in the right hand side of $(3.3 .6)_{p}$; therefore, if $\psi_{0}\left(=w_{0}\right), \psi_{1}, \ldots, \psi_{p-1}$ are known, by looking at the coefficients of $t^{p}$ in the both sides of $(3.3 .6)_{p}$ we can uniquely determine $\psi_{p}$. Moreover, if $\psi_{i}$ has the form $\psi_{i}\left(x, w_{0}, \ldots, w_{i}\right)$ for $i=1, \ldots, p-1$, we have the result that $\psi_{p}$ also has the form $\psi_{p}\left(x, w_{0}, \ldots, w_{p}\right)$. Thus, we have proved that the equation (3.3.3) has a unique solution and it has the form (3.3.2).

Next, let us show that the above solution $\psi\left(t, x, w_{0}, w_{1}, \ldots\right)$ satisfies the equation (3.3.4). To do so, it is enough to prove that

$$
u_{0} \equiv \sum_{i=0}^{p} \psi_{i}\left(x, \sum_{k=0}^{p-i} \phi_{k} t^{k}, \ldots, \sum_{k=0}^{p-i} D^{i}\left[\phi_{k}\right] t^{k}\right) t^{i} \quad \bmod O\left(t^{p+1}\right)
$$

(with $\phi_{0}=u_{0}$ and $\psi_{0}=w_{0}$ ) holds for all $p=0,1,2, \ldots$ Let us show this by induction on $p$. Our conditions are: $\psi_{0}=w_{0}, \psi_{1}=-\phi_{1}\left(x, w_{0}, w_{1}\right)$ and the relations $(3.3 .6)_{p}$ (for $\left.p \geq 1\right)$.

When $p=0$, the relation $(3.3 .7)_{0}$ is nothing but the equality $u_{0}=\psi_{0}\left(x, \phi_{0}\right)$ $=\phi_{0}$. When $p=1$, the right hand side of $(3.3 .7)_{1}$ is

$$
\begin{aligned}
& \psi_{0}\left(x, \phi_{0}+\phi_{1} t\right)+\psi_{1}\left(x, \phi_{0}, D\left[\phi_{0}\right]\right) t=\phi_{0}+\phi_{1} t+\psi_{1}\left(x, u_{0}, u_{1}\right) t \\
& =u_{0}+\left(\phi_{1}\left(x, u_{0}, u_{1}\right)+\psi_{1}\left(x, u_{0}, u_{1}\right)\right) t=u_{0}
\end{aligned}
$$


which is verified by $\phi_{0}=u_{0}$ and the definition of $\psi_{1}\left(x, u_{0}, u_{1}\right)$ : this proves $(3.3 .7)_{1}$.

Let $p \geq 2$ and suppose that $(3.3 .7)_{p-1}$ is already proved: then we have

$$
\begin{aligned}
u_{0} & \equiv \sum_{i=0}^{p-1} \psi_{i}\left(x, \sum_{k=0}^{p-1-i} \phi_{k} t^{k}, \ldots, \sum_{k=0}^{p-1-i} D^{i}\left[\phi_{k}\right] t^{k}\right) t^{i} \bmod O\left(t^{p}\right) \\
& \equiv \sum_{i=0}^{p-1} \psi_{i}\left(x, \sum_{k=0}^{p-1} \phi_{k} t^{k}, \ldots, \sum_{k=0}^{p-1} D^{i}\left[\phi_{k}\right] t^{k}\right) t^{i} \bmod O\left(t^{p}\right) .
\end{aligned}
$$

Since $D^{j}\left[u_{0}\right]=u_{j}$ holds, by applying $D^{j}$ to the both sides of the above equality and by using (2) of Lemma 3.1.5 we have

$$
\begin{aligned}
& u_{j} \equiv \sum_{i=0}^{p-1} D^{j}\left[\psi_{i}\right]\left(x, \sum_{k=0}^{p-1} \phi_{k} t^{k}, \ldots, \sum_{k=0}^{p-1} D^{i+j}\left[\phi_{k}\right] t^{k}\right) t^{i} \bmod O\left(t^{p}\right) \\
& \text { for } j=0,1,2, \ldots
\end{aligned}
$$

Since $(3.3 .6)_{p}$ is a known relation we have

$$
\begin{aligned}
& \sum_{i=1}^{p} \psi_{i}\left(x, w_{0}, \ldots, w_{i}\right) t^{i} \\
& \equiv-\sum_{k=1}^{p} \phi_{k}\left(x, \sum_{i=0}^{p-1} \psi_{i} t^{i}, \ldots, \sum_{i=0}^{p-1} D^{k}\left[\psi_{i}\right] t^{i}\right) t^{k} \bmod O\left(t^{p+1}\right)
\end{aligned}
$$

as functions with respect to the variables $\left(t, x, w_{0}, w_{1}, \ldots\right)$. By substituting

$$
w_{i}=\sum_{k=0}^{p-1} D^{i}\left[\phi_{k}\right]\left(x, u_{0}, \ldots, u_{k+i}\right) t^{k}, \quad i=0,1,2, \ldots
$$

into the both sides of (3.3.9) we have the relation

$$
\begin{aligned}
& \sum_{i=1}^{p} \psi_{i}\left(x, \sum_{k=0}^{p-1} \phi_{k} t^{k}, \ldots, \sum_{k=0}^{p-1} D^{i}\left[\phi_{k}\right] t^{k}\right) t^{i} \\
& \equiv-\sum_{k=1}^{p} \phi_{k}\left(x, \sum_{i=0}^{p-1} \psi_{i}\left(x, \sum_{k=0}^{p-1} \phi_{k} t^{k}, \ldots, \sum_{k=0}^{p-1} D^{i}\left[\phi_{k}\right] t^{k}\right) t^{i}, \ldots,\right. \\
&\left.\sum_{i=0}^{p-1} D^{k}\left[\psi_{i}\right]\left(x, \sum_{k=0}^{p-1} \phi_{k} t^{k}, \ldots, \sum_{k=0}^{p-1} D^{i+k}\left[\phi_{k}\right] t^{k}\right) t^{i}\right) t^{k}
\end{aligned}
$$

$\bmod O\left(t^{p+1}\right)$ 
as functions with respect to the variables $\left(t, x, u_{0}, u_{1}, \ldots\right)$. Therefore, by applying (3.3.8) to the right hand side of (3.3.10) we obtain

$$
\begin{aligned}
& \sum_{i=1}^{p} \psi_{i}\left(x, \sum_{k=0}^{p-1} \phi_{k} t^{k}, \ldots, \sum_{k=0}^{p-1} D^{i}\left[\phi_{k}\right] t^{k}\right) t^{i} \\
& \equiv-\sum_{k=1}^{p} \phi_{k}\left(x, u_{0}+O\left(t^{p}\right), \ldots, u_{k}+O\left(t^{p}\right)\right) t^{k} \bmod O\left(t^{p+1}\right) \\
& \quad \equiv-\sum_{k=1}^{p} \phi_{k}\left(x, u_{0}, \ldots, u_{k}\right) t^{k} \quad \bmod O\left(t^{p+1}\right)
\end{aligned}
$$

which immediately leads us to $(3.3 .7)_{p}$.

Thus, Lemma 3.3.2 is proved.

Proof of Proposition 3.3.1. Let $\psi\left(t, x, w_{0}, w_{1}, \ldots\right)$ be the solution of (3.3.3) in Lemma 3.3.2. Let us show the equivalence between the relations

$$
u_{j}=D^{j}[\psi]\left(t, x, w_{0}, w_{1}, \ldots\right), \quad j=0,1,2, \ldots
$$

and

$$
w_{j}=D^{j}[\phi]\left(t, x, u_{0}, u_{1}, \ldots\right), \quad j=0,1,2, \ldots
$$

Let us apply $D^{j}$ to the both sides of the equality (3.3.3); by (2) of Lemma 3.1 .5 we have

$$
w_{j}=D^{j}[\phi]\left(t, x, \psi, D[\psi], D^{2}[\psi], \ldots\right), \quad j=0,1,2, \ldots
$$

and therefore under the relation (3.3.11) we can get the relation (3.3.12). Similarly, by applying $D^{j}$ to the both sides of the equality (3.3.4) we have

$$
u_{j}=D^{j}[\psi]\left(t, x, \phi, D[\phi], D^{2}[\phi], \ldots\right), \quad j=0,1,2, \ldots
$$

and therefore under the relation (3.3.12) we can get the relation (3.3.11). This proves Proposition 3.3.1.

\section{$\S 3.4$. Composition of two couplings}

Let us consider three partial differential equations (A), (B) and

$$
\frac{\partial z}{\partial t}=H\left(t, x, z, \frac{\partial z}{\partial x}\right)
$$


(where $(t, x) \in \mathbb{C}^{2}$ are variables and $z=z(t, x)$ is the unknown function). The coupling equation $(\mathrm{A})$ and $(\mathrm{B})$ is

$$
\frac{\partial \phi}{\partial t}+\sum_{i \geq 0} D^{i}[F]\left(t, x, u_{0}, \ldots, u_{i+1}\right) \frac{\partial \phi}{\partial u_{i}}=G(t, x, \phi, D[\phi])
$$

and the coupling equation of $(\mathrm{B})$ and $(\mathrm{C})$ is

$$
\frac{\partial \eta}{\partial t}+\sum_{i \geq 0} D^{i}[G]\left(t, x, w_{0}, \ldots, w_{i+1}\right) \frac{\partial \eta}{\partial w_{i}}=H(t, x, \eta, D[\eta])
$$

(where $\eta=\eta\left(t, x, w_{0}, w_{1}, \ldots\right)$ is the unknown function). We have

Proposition 3.4.1. Let $\phi\left(t, x, u_{0}, u_{1}, u_{2}, \ldots\right)$ be a solution of (3.4.1), and let $\eta\left(t, x, w_{0}, w_{1}, w_{2}, \ldots\right)$ be a solution of (3.4.2). Then, the composition $\zeta=\eta\left(t, x, \phi, D[\phi], D^{2}[\phi], \ldots\right)$ is a solution of

$$
\frac{\partial \zeta}{\partial t}+\sum_{i \geq 0} D^{i}[F]\left(t, x, u_{0}, \ldots, u_{i+1}\right) \frac{\partial \zeta}{\partial u_{i}}=H(t, x, \zeta, D[\zeta])
$$

(which is the coupling equation of $(\mathrm{A})$ and $(\mathrm{C})$ ).

Proof. Set

$$
\zeta\left(t, x, u_{0}, u_{1}, u_{2}, \ldots\right)=\eta\left(t, x, \phi, D[\phi], D^{2}[\phi], \ldots\right)\left(=\eta_{\phi}\right) .
$$

Then, by Lemma 3.1.5, (3.4.1) and (3.4.2) we have

$$
\begin{aligned}
& \frac{\partial \zeta}{\partial t}+\sum_{i \geq 0} D^{i}[F] \frac{\partial \zeta}{\partial u_{i}} \\
& =\left(\frac{\partial \eta}{\partial t}\right)_{\phi}+\sum_{j \geq 0}\left(\frac{\partial \eta}{\partial w_{j}}\right)_{\phi} \frac{\partial D^{j}[\phi]}{\partial t}+\sum_{i \geq 0} D^{i}[F] \sum_{j \geq 0}\left(\frac{\partial \eta}{\partial w_{j}}\right)_{\phi} \frac{\partial D^{j}[\phi]}{\partial u_{i}} \\
& =\left(\frac{\partial \eta}{\partial t}\right)_{\phi}+\sum_{j \geq 0}\left(\frac{\partial \eta}{\partial w_{j}}\right)_{\phi}\left[\frac{\partial D^{j}[\phi]}{\partial t}+\sum_{i \geq 0} D^{i}[F] \frac{\partial D^{j}[\phi]}{\partial u_{i}}\right] \\
& =\left(\frac{\partial \eta}{\partial t}\right)_{\phi}+\sum_{j \geq 0}\left(\frac{\partial \eta}{\partial w_{j}}\right)_{\phi} D^{j}\left[\frac{\partial \phi}{\partial t}+\sum_{i \geq 0} D^{i}[F] \frac{\partial \phi}{\partial u_{i}}\right] \\
& =\left(\frac{\partial \eta}{\partial t}\right)_{\phi}+\sum_{j \geq 0}\left(\frac{\partial \eta}{\partial w_{j}}\right)_{\phi} D^{j}[G(t, x, \phi, D[\phi])] \\
& =\left(\frac{\partial \eta}{\partial t}\right)_{\phi}+\sum_{j \geq 0}\left(\frac{\partial \eta}{\partial w_{j}}\right)_{\phi} D^{j}[G]\left(t, x, \phi, D[\phi], \ldots, D^{1+j}[\phi]\right)
\end{aligned}
$$




$$
\begin{aligned}
& =\left(\frac{\partial \eta}{\partial t}+\sum_{j \geq 0} \frac{\partial \eta}{\partial w_{j}} D^{j}[G]\right)_{\phi}=(H(t, x, \eta, D[\eta]))_{\phi} \\
& =H\left(t, x, \eta_{\phi}, D[\eta]_{\phi}\right)=H\left(t, x, \eta_{\phi}, D\left[\eta_{\phi}\right]\right)=H(t, x, \zeta, D[\zeta]) .
\end{aligned}
$$

This proves Proposition 3.4.1.

By combining this with Definition 3.2.2, we can easily see

Proposition 3.4.2. If $(\mathrm{A})$ and $(\mathrm{B})$ are formally equivalent (resp. equivalent), and if $(\mathrm{B})$ and $(\mathrm{C})$ are formally equivalent (resp. equivalent), then $(\mathrm{A})$ and (C) are also formally equivalent (resp. equivalent).

As an application of Proposition 3.4.1, we will give another criterion of the reversibility of $\phi\left(t, x, u_{0}, u_{1}, \ldots\right)$. In the context of Section 3.1 , let $\phi\left(t, x, u_{0}, u_{1}\right.$, ...) be a solution of $(\Phi)$, and let $\psi\left(t, x, w_{0}, w_{1}, \ldots\right)$ be a solution of $(\Psi)$. Then, by Proposition 3.4.1 we see that the composition $\xi=\psi\left(t, x, \phi, D[\phi], D^{2}[\phi], \ldots\right)$ is a solution of

$$
\frac{\partial \xi}{\partial t}+\sum_{i \geq 0} D^{i}[F]\left(t, x, u_{0}, \ldots, u_{i+1}\right) \frac{\partial \xi}{\partial u_{i}}=F(t, x, \xi, D[\xi]),
$$

and the composition $\eta=\phi\left(t, x, \psi, D[\psi], D^{2}[\psi], \ldots\right)$ is a solution of

$$
\frac{\partial \eta}{\partial t}+\sum_{i \geq 0} D^{i}[G]\left(t, x, w_{0}, \ldots, w_{i+1}\right) \frac{\partial \eta}{\partial w_{i}}=G(t, x, \eta, D[\eta])
$$

It is easy to see that the equation (3.4.4) has a solution $\xi=u_{0}$; therefore, if the solution of

$$
\frac{\partial \xi}{\partial t}+\sum_{m \geq 0} D^{m}[F] \frac{\partial \xi}{\partial u_{m}}=F(t, x, \xi, D[\xi]),\left.\quad \xi\right|_{t=0}=u_{0}
$$

is unique and if $\left.\psi(t, x, \phi, D[\phi], \ldots)\right|_{t=0}=u_{0}$ holds, we have $u_{0}=\psi(t, x, \phi$, $D[\phi], \ldots)$. Similarly, if the uniqueness of the solution of

$$
\frac{\partial \eta}{\partial t}+\sum_{m \geq 0} D^{m}[G] \frac{\partial \eta}{\partial u_{m}}=G(t, x, \eta, D[\eta]),\left.\quad \eta\right|_{t=0}=w_{0}
$$

is valid and if $\left.\phi(t, x, \psi, D[\psi], \ldots)\right|_{t=0}=w_{0}$ holds, we have $w_{0}=\phi(t, x, \psi$, $D[\psi], \ldots)$. Thus, we obtain 
Proposition 3.4.3. Let $\phi\left(t, x, u_{0}, u_{1}, \ldots\right)$ be a solution of $(\Phi)$ with $\left.\phi\right|_{t=0}$ $=u_{0}$, and let $\psi\left(t, x, w_{0}, w_{1}, \ldots\right)$ be a solution of $(\Psi)$ with $\left.\psi\right|_{t=0}=w_{0}$. If the uniqueness of the solution is valid in two Cauchy problems (3.4.6) and (3.4.7), then we can conclude that the relation $w=\phi(t, x, u, \partial u / \partial x, \ldots)$ is reversible with respect to $u$ and $w$, and $\psi\left(t, x, w_{0}, w_{1}, \ldots\right)$ is the reverse function of $\phi\left(t, x, u_{0}, u_{1}, \ldots\right)$.

\section{$\S 4$. Equivalence of Two Partial Differential Equations}

In this section, we will give a concrete meaning to the formal theory in Section 3 and establish the equivalence of two partial differential equations.

Let $(t, x) \in \mathbb{C}_{t} \times \mathbb{C}_{x}$ be the variables, and let $F\left(t, x, z_{1}, z_{2}\right)$ be a holomorphic function defined in a neighborhood of the origin of $\mathbb{C}_{t} \times \mathbb{C}_{x} \times \mathbb{C}_{z_{1}} \times \mathbb{C}_{z_{2}}$. Let us consider the following partial differential equation

$$
\frac{\partial u}{\partial t}=F\left(t, x, u, \frac{\partial u}{\partial x}\right)
$$

and let us seek for a reduction to a simple form. As is seen in the case of ordinary differential equations (in [1]), it will be reasonable to treat the equation

$$
\frac{\partial w}{\partial t}=0
$$

as a candidate of the reduced form of (4.0.1). In order to justify this assertion, it is enough to discuss the following coupling equation

$$
\begin{gathered}
\frac{\partial \phi}{\partial t}+\sum_{m \geq 0} D^{m}[F]\left(t, x, u_{0}, \ldots, u_{m+1}\right) \frac{\partial \phi}{\partial u_{m}}=0, \quad \text { or } \\
\frac{\partial \psi}{\partial t}=F(t, x, \psi, D[\psi]) .
\end{gathered}
$$

In this section we denote by $\mathcal{R}(\mathbb{C} \backslash\{0\})$ the universal covering space of $\mathbb{C} \backslash\{0\}$, and we write $S_{\theta}=\{t \in \mathcal{R}(\mathbb{C} \backslash\{0\}) ;|\arg t|<\theta\}$ and $S_{\theta}(r)=\{t \in \mathcal{R}(\mathbb{C} \backslash$ $\{0\}) ; 0<|t| \leq r,|\arg t|<\theta\}$ for $\theta>0$ and $r>0$.

\section{$§ 4.1$. Formal solutions of $(\Phi)$ and $(\Psi)$}

First, let us look for a formal solution of the coupling equation $(\Phi)$ in the form

$$
\phi=u_{0}+\sum_{k \geq 1} \phi_{k}\left(x, u_{0}, \ldots, u_{k}\right) t^{k} \in \sum_{k \geq 0} \mathcal{O}_{R}\left[\left[u_{0}, \ldots, u_{k}\right]\right] t^{k}
$$

We have 
Proposition 4.1.1. The coupling equation $(\Phi)$ has a unique formal solution of the form (4.1.1). Moreover we have the following properties: i) $\phi_{1}\left(x, u_{0}, u_{1}\right)=-F\left(0, x, u_{0}, u_{1}\right)$, ii) $\phi_{k}\left(x, u_{0}, \ldots, u_{k}\right)($ for $k \geq 1)$ is a holomorphic function in a neighborhood of $\left\{\left(x, u_{0}, \ldots, u_{k}\right) \in \mathbb{C} \times \mathbb{C}^{k+1} ;|x| \leq R,\left|u_{0}\right| \leq\right.$ $\rho$ and $\left.\left|u_{1}\right| \leq \rho\right\}$ for some $R>0$ and $\rho>0$ which are independent of $k$, and iii) $\phi_{k}\left(x, u_{0}, \ldots, u_{k}\right)($ for $k \geq 2)$ is a polynomial with respect to $\left(u_{2}, \ldots, u_{k}\right)$.

Proof. Set $\phi_{0}=u_{0}$ and

$$
F\left(t, x, z_{1}, z_{2}\right)=\sum_{i \geq 0} F_{i}\left(x, z_{1}, z_{2}\right) t^{i}
$$

By substituting (4.1.1) into the equation $(\Phi)$ we have

$$
\sum_{k \geq 1} k \phi_{k} t^{k-1}+\sum_{i \geq 0} \sum_{j \geq 0} \sum_{0 \leq m \leq j} D^{m}\left[F_{i}\right]\left(x, u_{0}, \ldots, u_{m+1}\right) \frac{\partial \phi_{j}}{\partial u_{m}} t^{i+j}=0 .
$$

If we set $t=0$ in the above equality we see

$$
\phi_{1}=-F_{0}\left(x, u_{0}, u_{1}\right) \frac{\partial \phi_{0}}{\partial u_{0}}=-F_{0}\left(x, u_{0}, u_{1}\right)=-F\left(0, x, u_{0}, u_{1}\right),
$$

and also by looking at the coefficients of $t^{k}$ (with $k \geq 1$ ) we have the following recurrent formulas:

$$
\phi_{k+1}=-\frac{1}{k+1} \sum_{i+j=k} \sum_{0 \leq m \leq j} D^{m}\left[F_{i}\right]\left(x, u_{0}, \ldots, u_{m+1}\right) \frac{\partial \phi_{j}}{\partial u_{m}} .
$$

This proves that $\phi_{k}\left(x, u_{0}, \ldots, u_{k}\right)(k=2,3, \ldots)$ are determined uniquely by the formula (4.1.3) inductively on $k$. The other conditions follow from (4.1.3) and the definition of $D$.

Next, let us look for a formal solution of the coupling equation $(\Psi)$ in the form

$$
\psi=w_{0}+\sum_{k \geq 1} \psi_{k}\left(x, w_{0}, \ldots, w_{k}\right) t^{k} \in \sum_{k \geq 0} \mathcal{O}_{R}\left[\left[w_{0}, \ldots, w_{k}\right]\right] t^{k}
$$

We have

Proposition 4.1.2. The coupling equation $(\Psi)$ has a unique formal solution of the form (4.1.4). Moreover we have the following properties: i) $\psi_{1}\left(x, w_{0}, w_{1}\right)=F\left(0, x, w_{0}, w_{1}\right)$, ii) $\psi_{k}\left(x, w_{0}, \ldots, w_{k}\right)($ for $k \geq 1)$ is a holomorphic function in a neighborhood of $\left\{\left(x, w_{0}, \ldots, w_{k}\right) \in \mathbb{C} \times \mathbb{C}^{k+1} ;|x| \leq R,\left|w_{0}\right| \leq\right.$ $\rho$ and $\left.\left|w_{1}\right| \leq \rho\right\}$ for some $R>0$ and $\rho>0$ which are independent of $k$, and iii) $\psi_{k}\left(x, w_{0}, \ldots, w_{k}\right)($ for $k \geq 2)$ is a polynomial with respect to $\left(w_{2}, \ldots, w_{k}\right)$. 
Proof. Set $\psi_{0}=w_{0}$ and

$$
F\left(t, x, w_{0}+Y, w_{1}+Z\right)=\sum_{i, j, \alpha} a_{i, j, \alpha}\left(x, w_{0}, w_{1}\right) t^{i} Y^{j} Z^{\alpha} .
$$

Then, by substituting (4.1.4) into the equation $(\Psi)$ and by comparing the coefficients of $t^{k}$ in the both sides of the equation $(\Psi)$ we have the result that $\psi_{k}\left(x, w_{0}, \ldots, w_{k}\right)(k=1,2, \ldots)$ are determined uniquely by the following recurrent formulas: $\psi_{1}\left(x, w_{0}, w_{1}\right)=a_{0,0,0}\left(x, w_{0}, w_{1}\right)=F\left(0, x, w_{0}, w_{1}\right)$ and

$$
\begin{aligned}
\psi_{k+1}\left(x, w_{0}, \ldots, w_{k+1}\right)= & \frac{1}{k+1} \sum_{1 \leq i+j+\alpha \leq k} a_{i, j, \alpha}\left(x, w_{0}, w_{1}\right) \\
& \times \sum_{|l|+|m|=k-i} \psi_{l_{1}} \cdots \psi_{l_{j}} D\left[\psi_{m_{1}}\right] \cdots D\left[\psi_{m_{\alpha}}\right]
\end{aligned}
$$

(for $k \geq 1$ ), where $|l|=l_{1}+\cdots+l_{j}$ and $|m|=m_{1}+\cdots+m_{\alpha}$. This proves Proposition 4.1.2.

Let $\phi\left(t, x, u_{0}, u_{1}, \ldots\right)$ and $\psi\left(t, x, w_{0}, w_{1}, \ldots\right)$ be the formal solution in Propositions 4.1.1 and 4.1.2, respectively. Then, by Proposition 3.3.1 we see that the relation $w=\phi(t, x, u, \partial u / \partial x, \ldots)$ is reversible with respect to $u$ and $w$ and that $\psi\left(t, x, w_{0}, w_{1}, \ldots\right)$ is the reverse function of $\phi\left(t, x, u_{0}, u_{1}, \ldots\right)$. By Definition 3.2.2 we have

Theorem 4.1.3. The two equations (4.0.1) and (4.0.2) are formally equivalent.

\section{§4.2. Convergence of $\psi\left(t, x, w_{0}, w_{1}, \ldots\right)$}

In this section we will prove the convergence of the formal solution

$$
\psi=w_{0}+\sum_{k \geq 1} \psi_{k}\left(x, w_{0}, \ldots, w_{k}\right) t^{k}
$$

of the equation $(\Psi)$ in Proposition 4.1.2.

For $R_{1}>0, \varepsilon>0$ and $\eta>0$ we set

$$
\begin{aligned}
W_{k}\left(R_{1}, \varepsilon, \eta\right)=\{ & \left(x, w_{0}, \ldots, w_{k}\right) \in \mathbb{C} \times \mathbb{C}^{k+1} ;|x| \leq R_{1} \\
& \left.\left|w_{0}\right| \leq 0 ! \varepsilon / \eta^{0},\left|w_{1}\right| \leq 1 ! \varepsilon / \eta, \ldots,\left|w_{k}\right| \leq k ! \varepsilon / \eta^{k}\right\}
\end{aligned}
$$


$(k=1,2, \ldots)$. By Proposition 4.1 .2 and by taking $R_{1}>0, \varepsilon>0, \eta>0$ so that $R_{1}, \varepsilon$ and $\varepsilon / \eta$ are sufficiently small we may suppose that each $\psi_{k}\left(x, w_{0}, \ldots, w_{k}\right)$ is a holomorphic function on $W_{k}=W_{k}\left(R_{1}, \varepsilon, \eta\right)$ and so

$$
\left\|\psi_{k}\right\|_{W_{k}}=\max _{W_{k}}\left|\psi_{k}\left(x, w_{0}, \ldots, w_{k}\right)\right|<\infty, \quad k=1,2, \ldots
$$

We have

Proposition 4.2.1. Let $R_{1}>0$ be sufficiently small. Then, for any $\eta>0$ we can find an $\varepsilon>0$ such that the series

$$
\sum_{k \geq 1}\left\|\psi_{k}\right\|_{W_{k}} z^{k} \quad \text { with } W_{k}=W_{k}\left(R_{1}, \varepsilon, \eta\right)
$$

is convergent in a neighborhood of $z=0 \in \mathbb{C}$.

To prove this result, we suppose:

c-1) $F\left(t, x, z_{1}, z_{2}\right)$ is a holomorphic function in a neighborhood of $K=$ $\left\{\left(t, x, z_{1}, z_{2}\right) \in \mathbb{C}^{4} ;|t| \leq r,|x| \leq R_{0},\left|z_{1}\right| \leq \rho_{1},\left|z_{2}\right| \leq \rho_{2}\right\}$ for some $r>0, R_{0}>0, \rho_{1}>0$ and $\rho_{2}>0$, and

c-2) $\left|F\left(t, x, z_{1}, z_{2}\right)\right| \leq M$ on $K$.

Take any $0<\rho_{1}^{0}<\rho_{1}$ and $0<\rho_{2}^{0}<\rho_{2}$. Set $K_{0}=\left\{\left(x, w_{0}, w_{1}\right) \in \mathbb{C}^{3} ;|x| \leq\right.$ $\left.R_{0},\left|w_{0}\right| \leq \rho_{1}^{0},\left|w_{1}\right| \leq \rho_{2}^{0}\right\}$, and let $a_{i, j, \alpha}\left(x, w_{0}, w_{1}\right)\left((i, j, \alpha) \in \mathbb{N}^{3}\right)$ be the coefficients in (4.1.5): by Cauchy's inequality we have

$$
\left|a_{i, j, \alpha}\left(x, w_{0}, w_{1}\right)\right| \leq \frac{M}{r^{i}\left(\rho_{1}-\rho_{1}^{0}\right)^{j}\left(\rho_{2}-\rho_{2}^{0}\right)^{\alpha}} \quad \text { on } K_{0}
$$

for any $(i, j, \alpha) \in \mathbb{N}^{3}$.

Let $w(x)$ be a holomorphic function on $D_{R}=\{x \in \mathbb{C} ;|x| \leq R\}$ for some $R$ (with $0<R \leq R_{0}$ ) and suppose the condition

$$
\|w\|_{R} \leq \rho_{1}^{0} \quad \text { and } \quad\left\|\frac{\partial w}{\partial x}\right\|_{R} \leq \rho_{2}^{0}
$$

where we used the notation

$$
\|w\|_{R}=\max _{x \in D_{R}}|w(x)| .
$$

By (4.2.4) we have

$$
\left|a_{i, j, \alpha}(x, w, \partial w / \partial x)\right| \leq \frac{M}{r^{i}\left(\rho_{1}-\rho_{1}^{0}\right)^{j}\left(\rho_{2}-\rho_{2}^{0}\right)^{\alpha}} \quad \text { on } D_{R} .
$$

The following is the key result on the convergence of $\psi\left(t, x, w_{0}, w_{1}, \ldots\right)$. 
Proposition 4.2.2. $\quad$ Suppose the conditions c-1) and c-2). If $w(x)$ is a holomorphic function on $D_{R}$ for some $R$ (with $0<R \leq R_{0}$ ) and satisfies the condition (4.2.5) for some $\rho_{1}^{0}$ and $\rho_{2}^{0}$ (with $0<\rho_{1}^{0}<\rho_{1}$ and $\left.0<\rho_{2}^{0}<\rho_{2}\right)$, then the series

$$
\sum_{k \geq 1} \psi_{k}\left(x, w, \frac{\partial w}{\partial x}, \ldots, \frac{\partial^{k} w}{\partial x^{k}}\right) z^{k}
$$

is convergent at least in the domain

$$
\begin{aligned}
& \Omega=\left\{(x, z) \in \mathbb{C}^{2} ;|x|<R,\right. \\
&\left.\frac{|z|}{R-|x|}<\frac{r}{R+4 M r\left(\left(R /\left(\rho_{1}-\rho_{1}^{0}\right)\right)+\left(e /\left(\rho_{2}-\rho_{2}^{0}\right)\right)\right)}\right\} .
\end{aligned}
$$

Moreover, we have the following estimates:

$$
\begin{aligned}
& \sum_{k \geq 1}\left|\psi_{k}\left(x, w, \frac{\partial w}{\partial x}, \ldots, \frac{\partial^{k} w}{\partial x^{k}}\right)\right||z|^{k} \leq \frac{\rho_{1}-\rho_{1}^{0}}{2} \quad \text { on } \Omega \\
& \sum_{k \geq 1}\left|D\left[\psi_{k}\right]\left(x, w, \frac{\partial w}{\partial x}, \ldots, \frac{\partial^{k+1} w}{\partial x^{k+1}}\right)\right||z|^{k} \leq \frac{\rho_{2}-\rho_{2}^{0}}{2} \quad \text { on } \Omega .
\end{aligned}
$$

Before the proof of this result, we recall Nagumo's lemma:

Lemma 4.2.3 (Nagumo's lemma). If $f(x)$ is a holomorphic function on $\{x \in \mathbb{C} ;|x|<R\}$ and satisfies

$$
\|f\|_{s} \leq \frac{C}{(R-s)^{a}} \quad \text { for } 0<\forall s<R
$$

for some $C \geq 0$ and $a \geq 0$, we have

$$
\left\|\frac{\partial f}{\partial x}\right\|_{s} \leq \frac{(a+1) e C}{(R-s)^{a+1}} \quad \text { for } 0<\forall s<R .
$$

This was first proved by Nagumo [6] in a more general form; one can see a simple proof in the book [3] (Lemma 5.1.3 of [3]). In the proof of Proposition 4.2.2 this lemma will play an important role.

Proof of Proposition 4.2.2. By Proposition 4.1.2, we may assume that all the terms

$$
\psi_{k}\left(x, w, \frac{\partial w}{\partial x}, \ldots, \frac{\partial^{k} w}{\partial x^{k}}\right), \quad k=1,2, \ldots
$$

are holomorphic functions on $D_{R}$. 
We will prove the convergence of (4.2.7) by the method of majorant series. As an equation of majorant series, we adopt the following analytic functional equation with respect to $Y$ :

$$
\left\{\begin{array}{l}
Y=z \sum_{i+j+\alpha \geq 0} \frac{M}{r^{i}\left(\rho_{1}-\rho_{1}^{0}\right)^{j}\left(\rho_{2}-\rho_{2}^{0}\right)^{\alpha}}\left(\frac{R}{R-s}\right)^{i+j} z^{i} Y^{j}\left(\frac{e Y}{R-s}\right)^{\alpha} \\
Y(0)=0
\end{array}\right.
$$

where $s$ is a parameter with $0<s<R$. By the implicit function theorem we see that (4.2.11) has a unique holomorphic solution $Y=Y(z)$ in a neighborhood of $z=0 \in \mathbb{C}$. If we expand this into the form

$$
Y=\sum_{k \geq 1} Y_{k} z^{k}
$$

we easily see that the coefficients $Y_{k}(k=1,2, \ldots)$ are determined uniquely by the following recurrent formulas: $Y_{1}=M$ and

$$
\begin{aligned}
Y_{k+1}= & \sum_{1 \leq i+j+\alpha \leq k} \frac{M}{r^{i}\left(\rho_{1}-\rho_{1}^{0}\right)^{j}\left(\rho_{2}-\rho_{2}^{0}\right)^{\alpha}}\left(\frac{R}{R-s}\right)^{i+j} \\
& \times \sum_{|l|+|m|=k-i} Y_{l_{1}} \cdots Y_{l_{j}}\left(\frac{e Y_{m_{1}}}{R-s}\right) \cdots\left(\frac{e Y_{m_{\alpha}}}{R-s}\right)
\end{aligned}
$$

(for $g \geq 1$ ), where $|l|=l_{1}+\cdots+l_{j}$ and $|m|=m_{1}+\cdots+m_{\alpha}$. Moreover, by induction on $k$ we can see that $Y_{k}$ has the form

$$
Y_{k}=\frac{C_{k}}{(R-s)^{k-1}}, \quad k=1,2, \ldots
$$

where $C_{k} \geq 0(k=1,2, \ldots)$ are constants independent of the parameter $s$. We write $Y_{k}=Y_{k}(s)$ when we emphasize the fact that $Y_{k}$ depends on the parameter $s$ (with $0<s<R$ ).

The following lemma guarantees that $Y(z)=\sum_{k \geq 1} Y_{k}(s) z^{k}$ is a majorant series of (4.2.7).

Lemma 4.2.4. For any $k=1,2, \ldots$ we have:

$$
\begin{array}{ll}
(4.2 .13)_{k} & \left\|\psi_{k}\left(x, w, \frac{\partial w}{\partial x}, \ldots, \frac{\partial^{k} w}{\partial x^{k}}\right)\right\|_{s} \leq Y_{k}(s) \quad \text { for } 0<\forall s<R, \\
(4.2 .14)_{k} & \left\|D\left[\psi_{k}\right]\left(x, w, \frac{\partial w}{\partial x}, \ldots, \frac{\partial^{k+1} w}{\partial x^{k+1}}\right)\right\|_{s} \leq \frac{e Y_{k}(s)}{R-s} \quad \text { for } 0<\forall s<R .
\end{array}
$$


Proof of Lemma 4.2.4. We will prove this by induction on $k$. Since $\psi_{1}$ is defined by $\psi_{1}\left(x, w_{0}, w_{1}\right)=F\left(0, x, w_{0}, w_{1}\right)$, we have

$$
\left\|\psi_{1}\left(x, w, \frac{\partial w}{\partial x}\right)\right\|_{s} \leq\left\|F\left(0, x, w, \frac{\partial w}{\partial x}\right)\right\|_{R} \leq M=Y_{1}
$$

for $0<\forall s<R$. By Lemma 4.2.3 we have

$$
\left\|D\left[\psi_{1}\right]\left(x, w, \frac{\partial w}{\partial x}, \frac{\partial^{2} w}{\partial x^{2}}\right)\right\|_{s}=\left\|\left(\frac{\partial}{\partial x}\right) \psi_{1}\left(x, w, \frac{\partial w}{\partial x}\right)\right\|_{s} \leq \frac{e M}{R-s}=\frac{e Y_{1}}{R-s}
$$

for $0<\forall s<R$. This proves $(4.2 .13)_{1}$ and $(4.2 .14)_{1}$.

Next, let $k \geq 1$ and suppose that $(4.2 .13)_{p}$ and $(4.2 .14)_{p}$ are already proved for $p=1, \ldots, k$. Then, by (4.1.6), (4.2.6) and the induction hypothesis we have

$$
\begin{aligned}
& \left\|\psi_{k+1}\left(x, w, \frac{\partial w}{\partial x}, \ldots, \frac{\partial^{k+1} w}{\partial x^{k+1}}\right)\right\|_{s} \\
& \leq \frac{1}{k+1} \sum_{1 \leq i+j+\alpha \leq k} \frac{M}{r^{i}\left(\rho_{1}-\rho_{1}^{0}\right)^{j}\left(\rho_{2}-\rho_{2}^{0}\right)^{\alpha}} \\
& \quad \times \sum_{|l|+|m|=k-i} Y_{l_{1}} \cdots Y_{l_{j}}\left(\frac{e Y_{m_{1}}}{R-s}\right) \cdots\left(\frac{e Y_{m_{\alpha}}}{R-s}\right) .
\end{aligned}
$$

Therefore, by comparing this with (4.2.12) and by using $R /(R-s)>1$ we have

$$
\begin{aligned}
& \left\|\psi_{k+1}\left(x, w, \frac{\partial w}{\partial x}, \ldots, \frac{\partial^{k+1} w}{\partial x^{k+1}}\right)\right\|_{s} \\
& \leq \frac{1}{k+1} Y_{k+1}=\frac{1}{k+1} \frac{C_{k+1}}{(R-s)^{k}} \text { for } 0<\forall s<R:
\end{aligned}
$$

this yields $(4.2 .13)_{k+1}$. Moreover, by applying Lemma 4.2.3 to (4.2.15) we have

$$
\begin{aligned}
& \left\|D\left[\psi_{k+1}\right]\left(x, w, \frac{\partial w}{\partial x}, \ldots, \frac{\partial^{k+2} w}{\partial x^{k+2}}\right)\right\|_{s} \\
& =\left\|\left(\frac{\partial}{\partial x}\right) \psi_{k+1}\left(x, w, \frac{\partial w}{\partial x}, \ldots, \frac{\partial^{k+1} w}{\partial x^{k+1}}\right)\right\|_{s} \\
& \leq \frac{1}{k+1} \frac{(k+1) e C_{k+1}}{(R-s)^{k+1}}=\frac{e C_{k+1}}{(R-s)^{k+1}}=\frac{e Y_{k+1}}{R-s} \quad \text { for } 0<\forall s<R .
\end{aligned}
$$

This proves also $(4.2 .14)_{k+1}$.

Now, let us prove that the series (4.2.7) is convergent in the domain $\Omega$ in (4.2.8). By Lemma 4.2.4, for any fixed $s$ with $0<s<R$ we have

$$
\sum_{k \geq 1}\left\|\psi_{k}\left(x, w, \frac{\partial w}{\partial x}, \ldots, \frac{\partial^{k} w}{\partial x^{k}}\right)\right\|_{s}|z|^{k} \leq \sum_{k \geq 1} Y_{k}(s)|z|^{k}=Y(|z|)
$$


where $Y(z)$ is the unique solution of (4.2.11). Therefore, to prove the convergence of the series (4.2.7) on $\Omega$ it is sufficient to show that $Y(z)$ is convergent on $\Omega$, or equivalently that $Y(z)$ is holomorphic on $\Omega$.

Note that $Y(z) \gg 0$ and so

$$
\begin{aligned}
Y & =z \sum_{i+j+\alpha \geq 0} \frac{M}{r^{i}\left(\rho_{1}-\rho_{1}^{0}\right)^{j}\left(\rho_{2}-\rho_{2}^{0}\right)^{\alpha}}\left(\frac{R}{R-s}\right)^{i+j} z^{i} Y^{j}\left(\frac{e Y}{R-s}\right)^{\alpha} \\
& =M z \sum_{i+j+\alpha \geq 0}\left(\frac{R}{R-s} \frac{z}{r}\right)^{i}\left(\frac{R}{R-s} \frac{Y}{\rho_{1}-\rho_{1}^{0}}\right)^{j}\left(\frac{e}{R-s} \frac{Y}{\rho_{2}-\rho_{2}^{0}}\right)^{\alpha} \\
& \ll \frac{M z}{\left(1-\frac{R}{R-s} \frac{z}{r}\right)\left(1-\frac{R /\left(\rho_{1}-\rho_{1}^{0}\right)+e /\left(\rho_{2}-\rho_{2}^{0}\right)}{R-s} Y\right)},
\end{aligned}
$$

where $\sum_{i} a_{i} z^{i} \ll \sum_{i} b_{i} z^{i}$ means that $\left|a_{i}\right| \leq b_{i}$ holds for all $i$. Therefore, if we consider the equation

$$
\left\{\begin{array}{l}
Z=\frac{M z}{\left(1-\frac{R}{R-s} \frac{z}{r}\right)\left(1-\frac{R /\left(\rho_{1}-\rho_{1}^{0}\right)+e /\left(\rho_{2}-\rho_{2}^{0}\right)}{R-s} Z\right)} \\
Z(0)=0
\end{array}\right.
$$

we see that this equation has a unique holomorphic solution $Z(z)$ in a neighborhood of $z=0 \in \mathbb{C}$ and we have $Z(z) \gg Y(z)$. Moreover, by solving (4.2.16) concretely we have

$$
Z(z)=\frac{1}{2 \beta}\left(1-\sqrt{1-\frac{4 \beta M z}{1-\alpha z}}\right)
$$

with

$$
\alpha=\frac{R}{R-s} \frac{1}{r} \quad \text { and } \quad \beta=\frac{R /\left(\rho_{1}-\rho_{1}^{0}\right)+e /\left(\rho_{2}-\rho_{2}^{0}\right)}{R-s} .
$$

Thus, we conclude that $Z(z)$ is holomorphic in the domain

$$
\begin{aligned}
\Omega_{s} & =\left\{z \in \mathbb{C} ; \frac{4 \beta M|z|}{1-\alpha|z|}<1\right\} \\
& =\left\{z \in \mathbb{C} ; \frac{|z|}{R-s}<\frac{r}{R+4 M r\left(R /\left(\rho_{1}-\rho_{1}^{0}\right)+e /\left(\rho_{2}-\rho_{2}^{0}\right)\right)}\right\} .
\end{aligned}
$$

Since $Z(z) \gg Y(z)$ holds, we see that $Y(z)$ is also holomorphic on $\Omega_{s}$ and so the series

$$
\sum_{k \geq 1}\left\|\psi_{k}\left(x, w, \frac{\partial w}{\partial x}, \ldots, \frac{\partial^{k} w}{\partial x^{k}}\right)\right\|_{s}|z|^{k}
$$

is convergent in the domain $\Omega_{s}$. 
Since $s$ can be taken arbitrarily in $0<s<R$, we obtain the result that the series (4.2.7) is convergent in the domain

$$
\bigcup_{0<s<R}\left\{(x, z) \in \mathbb{C}^{2} ;|x| \leq s, z \in \Omega_{s}\right\}=\Omega .
$$

This proves the former half of Proposition 4.2.2.

Let us show the estimates (4.2.9) and (4.2.10). Since $Y(z) \ll Z(z)$, we have $Y(|z|) \leq Z(|z|)$; therefore, if $|x|=s$ we have

$$
\begin{gathered}
\sum_{k \geq 1}\left|\psi_{k}\left(x, w, \frac{\partial w}{\partial x}, \ldots, \frac{\partial^{k} w}{\partial x^{k}}\right)\right||z|^{k} \leq \sum_{k \geq 1}\left\|\psi_{k}\left(x, w, \frac{\partial w}{\partial x}, \ldots, \frac{\partial^{k} w}{\partial x^{k}}\right)\right\|_{s}|z|^{k} \\
\leq Y(|z|) \leq Z(|z|)=\frac{1}{2 \beta}\left(1-\sqrt{1-\frac{4 \beta M|z|}{1-\alpha|z|}}\right) \\
\leq \frac{1}{2 \beta}=\frac{(R-s)}{2\left(R /\left(\rho_{1}-\rho_{1}^{0}\right)+e /\left(\rho_{2}-\rho_{2}^{0}\right)\right)} \\
\leq \frac{R}{2 R /\left(\rho_{1}-\rho_{1}^{0}\right)}=\frac{\rho_{1}-\rho_{1}^{0}}{2} \text { on } \Omega
\end{gathered}
$$

this proves (4.2.9). Similarly, we have

$$
\begin{aligned}
\sum_{k \geq 1} \mid & \left.D\left[\psi_{k}\right]\left(x, w, \frac{\partial w}{\partial x}, \ldots, \frac{\partial^{k+1} w}{\partial x^{k+1}}\right)|| z\right|^{k} \\
& \leq \frac{e}{R-s} Y(|z|) \leq \frac{e}{R-s} Z(|z|)=\frac{e}{R-s} \frac{1}{2 \beta}\left(1-\sqrt{1-\frac{4 \beta M|z|}{1-\alpha|z|}}\right) \\
& \leq \frac{e}{R-s} \frac{1}{2 \beta}=\frac{e}{2\left(R /\left(\rho_{1}-\rho_{1}^{0}\right)+e /\left(\rho_{2}-\rho_{2}^{0}\right)\right)} \\
& \leq \frac{e}{2 e /\left(\rho_{2}-\rho_{2}^{0}\right)}=\frac{\rho_{2}-\rho_{2}^{0}}{2} \text { on } \Omega
\end{aligned}
$$

this proves (4.2.10).

Thus, all the parts of Proposition 4.2.2 are proved.

For $a(x)=\sum_{i \geq 0} a_{i} x^{i}$ we write $|a|(x)=\sum_{i \geq 0}\left|a_{i}\right| x^{i} \gg 0$; for $a(x, y)=$ $\sum_{i, j} a_{i, j} x^{i} y^{j}$ we write $|a|(x, y)=\sum_{i, j}\left|a_{i, j}\right| x^{i} y^{j} \gg 0$; and so on.

Let $F\left(t, x, z_{1}, z_{2}\right)$ be as before, let $|F|\left(t, x, z_{1}, z_{2}\right)$ be defined as above, and let us consider

$$
\frac{\partial \psi^{+}}{\partial t}=|F|\left(t, x, \psi^{+}, D\left[\psi^{+}\right]\right)
$$


It is clear by Proposition 4.1.2 that $\left(\Psi^{+}\right)$has a unique formal solution $\psi^{+}(t, x$, $\left.w_{0}, w_{1}, \ldots\right)$ of the form

$$
\psi^{+}=w_{0}+\sum_{k \geq 1} \psi_{k}^{+}\left(x, w_{0}, \ldots, w_{k}\right) t^{k} \in \sum_{k \geq 0} \mathcal{O}_{R}\left[\left[w_{0}, \ldots, w_{k}\right]\right] t^{k}
$$

and that $\psi_{k}^{+}\left(x, w_{0}, \ldots, w_{k}\right) \gg 0$ holds for all $k=1,2, \ldots$ By Proposition 4.2 .2 we have

Proposition 4.2.5. Suppose that $|F|\left(t, x, z_{1}, z_{2}\right)$ satisfies the conditions $\mathrm{c}-1)$ and $\mathrm{c}-2)$ with $F$ and $M$ replaced by $|F|$ and $M^{+}$, respectively. If $w(x)$ is a holomorphic function on $D_{R}$ for some $R$ (with $0<R \leq R_{0}$ ) and satisfies the condition (4.2.5) for some $\rho_{1}^{0}$ and $\rho_{2}^{0}$ (with $0<\rho_{1}^{0}<\rho_{1}$ and $\left.0<\rho_{2}^{0}<\rho_{2}\right)$, then the series

$$
\sum_{k \geq 1} \psi_{k}^{+}\left(x, w, \frac{\partial w}{\partial x}, \ldots, \frac{\partial^{k} w}{\partial x^{k}}\right) z^{k}
$$

is convergent at least in the domain

$$
\begin{aligned}
\Omega^{+}=\left\{(x, z) \in \mathbb{C}^{2} ;|x|<R,\right. \\
\left.\quad \frac{|z|}{R-|x|}<\frac{r}{R+4 M^{+} r\left(R /\left(\rho_{1}-\rho_{1}^{0}\right)+e /\left(\rho_{2}-\rho_{2}^{0}\right)\right)}\right\} .
\end{aligned}
$$

Moreover, we have the following estimates:

$$
\begin{aligned}
& \sum_{k \geq 1}\left|\psi_{k}^{+}\left(x, w, \frac{\partial w}{\partial x}, \ldots, \frac{\partial^{k} w}{\partial x^{k}}\right)\right||z|^{k} \leq \frac{\rho_{1}-\rho_{1}^{0}}{2} \quad \text { on } \Omega^{+} \\
& \sum_{k \geq 1}\left|D\left[\psi_{k}^{+}\right]\left(x, w, \frac{\partial w}{\partial x}, \ldots, \frac{\partial^{k+1} w}{\partial x^{k+1}}\right)\right||z|^{k} \leq \frac{\rho_{2}-\rho_{2}^{0}}{2} \quad \text { on } \Omega^{+} .
\end{aligned}
$$

By using this proposition, let us give a proof of Proposition 4.2.1.

Proof of Proposition 4.2.1. Let $r>0, R_{0}>0, \rho_{1}>0$ and $\rho_{2}>0$ be as in Proposition 4.2.5, and let $0<R \leq R_{0}, 0<\rho_{1}^{0}<\rho_{1}$ and $0<\rho_{2}^{0}<\rho_{2}$. Take $a>0$ and $L>R$ such that

$$
\frac{a}{L-R} \leq \rho_{1}^{0} \quad \text { and } \quad \frac{a}{(L-R)^{2}} \leq \rho_{2}^{0}
$$

hold. Set

$$
w(x)=\frac{a}{L-x} .
$$


Then, we see that $w(x)$ is a holomorphic function on $D_{R}$ and

$$
\frac{\partial^{k} w}{\partial x^{k}}=\frac{k ! a}{(L-x)^{k+1}}, \quad k=0,1,2, \ldots
$$

and so we have $\|w\|_{R} \leq \rho_{1}^{0}$ and $\|\partial w / \partial x\|_{R} \leq \rho_{2}^{0}$. Hence, by Proposition 4.2.5 and its proof we see: for any fixed $s$ with $0<s<R$ the series

$$
\sum_{k \geq 1}\left\|\psi_{k}^{+}\left(x, w, \frac{\partial w}{\partial x}, \ldots, \frac{\partial^{k} w}{\partial x^{k}}\right)\right\|_{s} z^{k}
$$

is convergent in the domain

$$
\Omega_{s}^{+}=\left\{z \in \mathbb{C} ; \frac{|z|}{R-s}<\frac{r}{R+4 M^{+} r\left(R /\left(\rho_{1}-\rho_{1}^{0}\right)+e /\left(\rho_{2}-\rho_{2}^{0}\right)\right)}\right\}
$$

Since $\psi^{+}\left(t, x, w_{0}, \ldots, w_{k}\right) \gg 0$ holds, we have

$$
\begin{aligned}
\| \psi_{k}^{+} & \left(x, w, \frac{\partial w}{\partial x}, \ldots, \frac{\partial^{k} w}{\partial x^{k}}\right) \|_{s} \\
& =\left\|\psi_{k}^{+}\left(x, \frac{0 ! a}{(L-x)}, \frac{1 ! a}{(L-x)^{2}}, \ldots, \frac{k ! a}{(L-x)^{k+1}}\right)\right\|_{s} \\
& =\psi_{k}^{+}\left(s, \frac{0 ! a}{(L-s)}, \frac{1 ! a}{(L-s)^{2}}, \ldots, \frac{k ! a}{(L-s)^{k+1}}\right) ;
\end{aligned}
$$

therefore, if we set $\varepsilon=a /(L-s), \eta=L-s$ and

$$
\begin{aligned}
W_{k}(s, \varepsilon, \eta)=\{ & \left(x, w_{0}, \ldots, w_{k}\right) \in \mathbb{C} \times \mathbb{C}^{k+1} ;|x| \leq s \\
& \left.\left|w_{0}\right| \leq 0 ! \varepsilon / \eta^{0},\left|w_{1}\right| \leq 1 ! \varepsilon / \eta, \ldots,\left|w_{k}\right| \leq k ! \varepsilon / \eta^{k}\right\},
\end{aligned}
$$

we have

$$
\begin{aligned}
\left\|\psi_{k}\right\|_{W_{k}} & \leq\left\|\psi_{k}^{+}\right\|_{W_{k}}=\psi_{k}^{+}\left(s, \frac{0 ! \varepsilon}{\eta^{0}}, \frac{1 ! \varepsilon}{\eta}, \ldots, \frac{k ! \varepsilon}{\eta^{k}}\right) \\
& =\psi_{k}^{+}\left(s, \frac{0 ! a}{(L-s)}, \frac{1 ! a}{(L-s)^{2}}, \ldots, \frac{k ! a}{(L-s)^{k+1}}\right) \\
& =\left\|\psi_{k}^{+}\left(x, w, \frac{\partial w}{\partial x}, \ldots, \frac{\partial^{k} w}{\partial x^{k}}\right)\right\|_{s} .
\end{aligned}
$$

Thus, by the convergence of (4.2.22) we obtain the result that the series

$$
\sum_{k \geq 1}\left\|\psi_{k}\right\|_{W_{k}} z^{k} \quad \text { with } W_{k}=W_{k}(s, \varepsilon, \eta)
$$

is convergent on $\Omega_{s}^{+}$. 
Now, let us justify the assertion of Proposition 4.2.1. Take any $0<R_{1}<$ $R_{0}$ and fix it. For any $\eta>0$ we take $\varepsilon$ so that

$$
0<\varepsilon \leq \min \left\{\rho_{1}^{0} / 2, \rho_{2}^{0} \eta / 4\right\}
$$

Set $L=R_{1}+\eta, a=\varepsilon\left(L-R_{1}\right)$ and $R=\min \left\{R_{0}, R_{1}+\eta / 2\right\}$; then we have $\eta=L-R_{1}, \varepsilon=a /\left(L-R_{1}\right), L-R \geq L-R_{1}-\eta / 2=\eta / 2$,

$$
\begin{gathered}
\frac{a}{L-R}=\frac{\varepsilon\left(L-R_{1}\right)}{L-R} \leq \frac{\varepsilon \eta}{\eta / 2}=2 \varepsilon \leq \rho_{1}^{0}, \\
\frac{a}{(L-R)^{2}}=\frac{\varepsilon\left(L-R_{1}\right)}{(L-R)^{2}} \leq \frac{\varepsilon \eta}{(\eta / 2)^{2}}=\frac{4 \varepsilon}{\eta} \leq \rho_{2}^{0} .
\end{gathered}
$$

Thus, by setting $s=R_{1}$ we can conclude that the series (4.2.24) with $s=R_{1}$ is convergent in the domain $\Omega_{R_{1}}^{+}$(with $R=\min \left\{R_{0}, R_{1}+\eta / 2\right\}$ ). This proves Proposition 4.2.1.

By the proof we have

Proposition 4.2.6. Suppose that $|F|\left(t, x, z_{1}, z_{2}\right)$ satisfies the conditions c-1) and c-2) with $F$ and $M$ replaced by $|F|$ and $M^{+}$, respectively. Let $0<$ $\rho_{1}^{0}<\rho_{1}$ and $0<\rho_{2}^{0}<\rho_{2}$. Then, for any $0<R_{1}<R_{0}, \eta>0$ and $0<\varepsilon \leq$ $\min \left\{\rho_{1}^{0} / 2, \rho_{2}^{0} \eta / 4\right\}$ the series

$$
\sum_{k \geq 1}\left\|\psi_{k}^{+}\right\|_{W_{k}} z^{k} \text { with } W_{k}=W_{k}\left(R_{1}, \varepsilon, \eta\right)
$$

is convergent in the domain $\Omega_{R_{1}}^{+}\left(\right.$in $(4.2 .23)$ with $s=R_{1}$ and $R=\min \left\{R_{0}\right.$, $\left.\left.R_{1}+\eta / 2\right\}\right)$. Moreover, we have the following estimate:

$$
\sum_{k \geq 1}\left\|\psi_{k}^{+}\right\|_{W_{k}}|z|^{k} \leq \frac{\rho_{1}-\rho_{1}^{0}}{2} \quad \text { on } \Omega_{R_{1}}^{+} .
$$

The following corollary explains how to use this result.

Corollary 4.2.7. Let $\psi\left(t, x, w_{0}, w_{1}, \ldots\right)$ be the unique solution of $(\Psi)$ of the form (4.2.1), and let $w(t, x)$ be a holomorphic function on $S_{\theta}(r) \times D_{R}$ for some $\theta>0, r>0$ and $R>0$. If

$$
\sup _{S_{\theta}(r) \times D_{R}}|w(t, x)|<\min \left\{\rho_{1} / 2, \rho_{2} R / 4\right\}
$$

holds, the function $\psi(t, x, w, \partial w / \partial x, \ldots)$ is convergent and defines a holomorphic function on $S_{\theta}\left(r_{1}\right) \times D_{R_{1}}$ for some $r_{1}>0$ and $R_{1}>0$. 
Proof. By the assumption we can take $\varepsilon>0,0<\eta<R, 0<\rho_{1}^{0}<\rho_{1}$ and $0<\rho_{2}^{0}<\rho_{2}$ such that

$$
\sup _{S_{\theta}(r) \times D_{R}}|w(t, x)| \leq \varepsilon \leq \min \left\{\rho_{1}^{0} / 2, \rho_{2}^{0} \eta / 4\right\} .
$$

Then, by Cauchy's inequality we have

$$
\left\|\frac{\partial^{k} w}{\partial x^{k}}(t)\right\|_{R-\eta} \leq \frac{k ! \varepsilon}{\eta^{k}} \text { on } S_{\theta}(r), k=0,1,2, \ldots
$$

and therefore by setting $R_{1}=R-\eta$ and $W_{k}=W_{k}\left(R_{1}, \varepsilon, \eta\right)(k=1,2, \ldots)$ we have

$$
\begin{aligned}
& \sum_{k \geq 1}\left\|\psi_{k}\left(x, w, \frac{\partial w}{\partial x}, \ldots, \frac{\partial^{k} w}{\partial x^{k}}\right)\right\|_{R_{1}}|t|^{k} \\
& \quad \leq \sum_{k \geq 1}\left\|\psi_{k}\right\|_{W_{k}}|t|^{k} \leq \sum_{k \geq 1}\left\|\psi_{k}^{+}\right\|_{W_{k}}|t|^{k}<\infty, \quad \text { if } t \in S_{\theta}(r) \cap \Omega_{R_{1}}^{+}
\end{aligned}
$$

This proves Corollary 4.2.7.

Remark. Of course, this corollary can be verified directly by Proposition 4.2.2.

\section{$\S 4.3$. Convergence of $\phi\left(t, x, u_{0}, u_{1}, \ldots\right)$}

In this section we will prove the convergence of the formal solution

$$
\phi=u_{0}+\sum_{k \geq 1} \phi_{k}\left(x, u_{0}, \ldots, u_{k}\right) t^{k}
$$

of the equation $(\Phi)$ in Proposition 4.1.1.

For $R_{1}>0, \varepsilon>0$ and $\eta>0$ we set

$$
\begin{aligned}
U_{k}\left(R_{1}, \varepsilon, \eta\right)=\{ & \left(x, u_{0}, \ldots, u_{k}\right) \in \mathbb{C} \times \mathbb{C}^{k+1} ;|x| \leq R_{1}, \\
& \left.\left|u_{0}\right| \leq 0 ! \varepsilon / \eta^{0},\left|u_{1}\right| \leq 1 ! \varepsilon / \eta, \ldots,\left|u_{k}\right| \leq k ! \varepsilon / \eta^{k}\right\}
\end{aligned}
$$

$(k=1,2, \ldots)$. By Proposition 4.1 .1 and by taking $R_{1}>0, \varepsilon>0, \eta>0$ so that $R_{1}, \varepsilon$ and $\varepsilon / \eta$ are sufficiently small we may suppose that each $\phi_{k}\left(x, u_{0}, \ldots, u_{k}\right)$ is a holomorphic function on $U_{k}=U_{k}\left(R_{1}, \varepsilon, \eta\right)$ and so

$$
\left\|\phi_{k}\right\|_{U_{k}}=\max _{U_{k}}\left|\phi_{k}\left(x, u_{0}, \ldots, u_{k}\right)\right|<\infty, \quad k=1,2, \ldots
$$

We have 
Proposition 4.3.1. Let $R_{1}>0$ be sufficiently small. Then, for any $\eta>0$ we can find an $\varepsilon>0$ such that the series

$$
\sum_{k \geq 1}\left\|\phi_{k}\right\|_{U_{k}} z^{k} \quad \text { with } U_{k}=U_{k}\left(R_{1}, \varepsilon, \eta\right)
$$

is convergent in a neighborhood of $z=0 \in \mathbb{C}$.

To prove this, we consider

$$
\frac{\partial \psi^{+}}{\partial t}=|F|\left(t, x, \psi^{+}, D\left[\psi^{+}\right]\right)
$$

let

$$
\psi^{+}=w_{0}+\sum_{k \geq 1} \psi_{k}^{+}\left(x, w_{0}, \ldots, w_{k}\right) t^{k}
$$

be the unique solution of this equation. Then, Proposition 4.3.1 follows easily from Proposition 4.2.6, Lemma 4.3.2 given below and Stirling's formula

$$
\sqrt{2 \pi} x^{x+1 / 2} e^{-x} \leq \Gamma(1+x) \leq \sqrt{2 \pi} x^{x+1 / 2} e^{-x+1} \quad \text { for } x>0 .
$$

Lemma 4.3.2. For any $k=0,1,2, \ldots$ we have

$(4.3 .5)_{k} \quad\left|\phi_{k}\right|\left(x, \psi^{+}, \ldots, D^{k}\left[\psi^{+}\right]\right) \ll \sum_{q \geq 0} \frac{(k+q)^{k}}{k !} \psi_{k+q}^{+}\left(x, w_{0}, \ldots, w_{k+q}\right) t^{q}$

(with $\phi_{0}=u_{0}$ and $\left.\psi_{0}^{+}=w_{0}\right)$. In particular, by setting $t=0$ we have

$$
\left|\phi_{k}\right|\left(x, w_{0}, \ldots, w_{k}\right) \ll \frac{k^{k}}{k !} \psi_{k}^{+}\left(x, w_{0}, \ldots, w_{k}\right) .
$$

Proof. When $k=0$, we have $\phi_{0}\left(x, u_{0}\right)=u_{0}$ and

$$
\left|\phi_{0}\right|\left(x, \psi^{+}\right)=\psi^{+}=\sum_{q \geq 0} \psi_{q}^{+} t^{q}
$$

this proves $(4.3 .5)_{0}$. When $k=1$, we have $\phi_{1}\left(x, u_{0}, u_{1}\right)=-F\left(0, x, u_{0}, u_{1}\right)$ and so

$$
\begin{aligned}
& \left|\phi_{1}\right|\left(x, \psi^{+}, D\left[\psi^{+}\right]\right)=|F|\left(0, x, \psi^{+}, D\left[\psi^{+}\right]\right) \\
& \ll|F|\left(t, x, \psi^{+}, D\left[\psi^{+}\right]\right) \\
& \quad=\frac{\partial \psi^{+}}{\partial t}=\frac{\partial}{\partial t} \sum_{p \geq 0} \psi_{p}^{+} t^{p}=\sum_{q \geq 0}(1+q) \psi_{1+q}^{+} t^{q}
\end{aligned}
$$


this proves $(4.3 .5)_{1}$.

Let $k \geq 1$ and suppose that $(4.3 .5)_{p}$ is already proved for $p=0,1, \ldots, k$. Then, by (4.1.3) we have

$$
\left|\phi_{k+1}\right| \ll \frac{1}{k+1} \sum_{i+j=k} \sum_{0 \leq m \leq j} D^{m}\left[\left|F_{i}\right|\right]\left(x, u_{0}, \ldots, u_{m+1}\right) \frac{\partial\left|\phi_{j}\right|}{\partial u_{m}}
$$

and so

$$
\begin{array}{r}
\left|\phi_{k+1}\right|\left(x, \psi^{+}, \ldots, D^{k+1}\left[\psi^{+}\right]\right) t^{k} \\
\ll \frac{1}{k+1} \sum_{i+j=k} \sum_{0 \leq m \leq j} D^{m}\left[\left|F_{i}\right|\right]\left(x, \psi^{+}, \ldots, D^{m+1}\left[\psi^{+}\right]\right) t^{i} \\
\times \frac{\partial\left|\phi_{j}\right|}{\partial u_{m}}\left(x, \psi^{+}, \ldots, D^{j}\left[\psi^{+}\right]\right) t^{j} .
\end{array}
$$

Since we have by (2) of Lemma 3.1.5

$$
\begin{aligned}
& D^{m}\left[\left|F_{i}\right|\right]\left(x, \psi^{+}, \ldots, D^{m+1}\left[\psi^{+}\right]\right) t^{i}=D^{m}\left[\left|F_{i}\right|\left(x, \psi^{+}, D\left[\psi^{+}\right]\right) t^{i}\right] \\
& \ll D^{m}\left[|F|\left(t, x, \psi^{+}, D\left[\psi^{+}\right]\right)\right]=D^{m}\left[\frac{\partial \psi^{+}}{\partial t}\right]=\frac{\partial D^{m}\left[\psi^{+}\right]}{\partial t},
\end{aligned}
$$

by applying this to (4.3.7) we have

$$
\begin{aligned}
& \left|\phi_{k+1}\right|\left(x, \psi^{+}, \ldots, D^{k+1}\left[\psi^{+}\right]\right) t^{k} \\
& \ll \frac{1}{k+1} \sum_{0 \leq j \leq k} \sum_{0 \leq m \leq j} \frac{\partial D^{m}\left[\psi^{+}\right]}{\partial t} \frac{\partial\left|\phi_{j}\right|}{\partial u_{m}}\left(x, \psi^{+}, \ldots, D^{j}\left[\psi^{+}\right]\right) t^{j} \\
& =\frac{1}{k+1} \sum_{0 \leq j \leq k} \frac{\partial}{\partial t}\left(\left|\phi_{j}\right|\left(x, \psi^{+}, \ldots, D^{j}\left[\psi^{+}\right]\right)\right) \times t^{j} .
\end{aligned}
$$

Thus, by the induction hypothesis we obtain

$$
\begin{aligned}
& \left|\phi_{k+1}\right|\left(x, \psi^{+}, \ldots, D^{k+1}\left[\psi^{+}\right]\right) t^{k} \\
& \ll \frac{1}{k+1} \sum_{0 \leq j \leq k} \frac{\partial}{\partial t}\left(\sum_{q \geq 0} \frac{(j+q)^{j}}{j !} \psi_{j+q}^{+}\left(x, w_{0}, \ldots, w_{j+q}\right) t^{q}\right) \times t^{j} \\
& =\frac{1}{k+1} \sum_{0 \leq j \leq k} \sum_{q \geq 1} \frac{(j+q)^{j}}{j !} q \psi_{j+q}^{+}\left(x, w_{0}, \ldots, w_{j+q}\right) t^{q-1+j} \\
& =\frac{1}{k+1} \sum_{0 \leq j \leq k} \sum_{p \geq j} \frac{(p+1)^{j}}{j !}(p+1-j) \psi_{p+1}^{+}\left(x, w_{0}, \ldots, w_{p+1}\right) t^{p} .
\end{aligned}
$$


Since the degree of each term of the left hand side of (4.3.9) in $t$ is greater than or equal to $k$, by looking at the terms whose degree in $t$ is greater than or equal to $k$ and then by canceling $t^{k}$ from the both sides we obtain

$$
\begin{aligned}
& \left|\phi_{k+1}\right|\left(x, \psi^{+}, \ldots, D^{k+1}\left[\psi^{+}\right]\right) \\
& \ll \frac{1}{k+1} \sum_{0 \leq j \leq k} \sum_{p \geq k} \frac{(p+1)^{j}}{j !}(p+1-j) \psi_{p+1}^{+}\left(x, w_{0}, \ldots, w_{p+1}\right) t^{p-k} \\
& =\frac{1}{k+1} \sum_{q \geq 0} \sum_{0 \leq j \leq k} \frac{(k+1+q)^{j}}{j !}(k+1+q-j) \\
& \times \psi_{k+1+q}^{+}\left(x, w_{0}, \ldots, w_{k+1+q}\right) t^{q} \\
& =\frac{1}{k+1} \sum_{q \geq 0}\left[\sum_{0 \leq j \leq k}\left(\frac{(k+1+q)^{j+1}}{j !}-\frac{j(k+1+q)^{j}}{j !}\right)\right] \\
& =\frac{1}{k+1} \sum_{q \geq 0} \frac{(k+1+q)^{k+1}}{k !} \psi_{k+1+q}^{+}\left(x, w_{0}, \ldots, w_{k+1+q}\right) t^{q} \\
& =\sum_{q \geq 0} \frac{(k+1+q)^{k+1}}{(k+1) !} \psi_{k+1+q}^{+}\left(x, w_{0}, \ldots, w_{k+1+q}\right) t^{q} ;
\end{aligned}
$$

this proves $(4.3 .5)_{k+1}$.

Since $k^{k} / k ! \leq e^{k} / \sqrt{2 \pi}$ holds for any $k=1,2, \ldots$, we have the following precise form of Proposition 4.3.1:

Proposition 4.3.3. Suppose that $|F|\left(t, x, z_{1}, z_{2}\right)$ satisfies the conditions c-1) and c-2) with $F$ and $M$ replaced by $|F|$ and $M^{+}$, respectively. Let $0<$ $\rho_{1}^{0}<\rho_{1}$ and $0<\rho_{2}^{0}<\rho_{2}$. Then, for any $0<R_{1}<R_{0}, \eta>0$ and $0<\varepsilon \leq$ $\min \left\{\rho_{1}^{0} / 2, \rho_{2}^{0} \eta / 4\right\}$ the series

$$
\sum_{k \geq 1}\left\|\phi_{k}\right\|_{U_{k}} z^{k} \text { with } U_{k}=U_{k}\left(R_{1}, \varepsilon, \eta\right)
$$

is convergent in the domain

$$
\begin{aligned}
& \Omega_{R_{1}, \eta}^{*}=\left\{z \in \mathbb{C} ; \frac{e|z|}{R-R_{1}}<\frac{r}{R+4 M^{+} r\left(R /\left(\rho_{1}-\rho_{1}^{0}\right)+e /\left(\rho_{2}-\rho_{2}^{0}\right)\right)}\right\} \\
& \left(\text { with } R=\min \left\{R_{0}, R_{1}+\eta / 2\right\}\right) .
\end{aligned}
$$

Moreover, we have the following estimate:

$$
\sum_{k \geq 1}\left\|\phi_{k}\right\|_{U_{k}}|z|^{k} \leq \frac{\rho_{1}-\rho_{1}^{0}}{2} \quad \text { on } \Omega_{R_{1}, \eta}^{*}
$$


Corollary 4.3.4. Let $\phi\left(t, x, u_{0}, u_{1}, \ldots\right)$ be the unique solution of $(\Phi)$ of the form (4.3.1), and let $u(t, x)$ be a holomorphic function on $S_{\theta}(r) \times D_{R}$ for some $\theta>0, r>0$ and $R>0$. If

$$
\sup _{S_{\theta}(r) \times D_{R}}|u(t, x)|<\min \left\{\rho_{1} / 2, \rho_{2} R / 4\right\}
$$

holds, the function $\phi(t, x, u, \partial u / \partial x, \ldots)$ is convergent and defines a holomorphic function on $S_{\theta}\left(r_{1}\right) \times D_{R_{1}}$ for some $r_{1}>0$ and $R_{1}>0$.

\section{§4.4. Equivalence of (4.0.1) and (4.0.2)}

Now, let us establish the equivalence of two equations (4.0.1) and (4.0.2). Let $r>0, R>0, \rho_{1}>0$ and $\rho_{2}>0$. We suppose:

$$
\begin{aligned}
& F\left(t, x, z_{1}, z_{2}\right) \text { is a holomorphic function on }\left\{\left(t, x, z_{1}, z_{2}\right) \in \mathbb{C}^{4} ;\right. \\
& \left.|t|<r,|x|<R,\left|z_{1}\right|<\rho_{1},\left|z_{2}\right|<\rho_{2}\right\} .
\end{aligned}
$$

Definition 4.4.1. (1) We denote by $\mathscr{X}$ the set of all the functions $w(t, x)$ satisfying the following properties:

i) $w(t, x)$ is a holomorphic function on $S_{\theta}\left(r_{1}\right) \times D_{R_{1}}$ for some $\theta>0$, $0<r_{1}<r$ and $0<R_{1}<R$, and

ii) $|w(t, x)| \leq \rho_{1}^{0}$ and $|(\partial w / \partial x)(t, x)| \leq \rho_{2}^{0}$ hold on $S_{\theta}\left(r_{1}\right) \times D_{R_{1}}$ for some $0<\rho_{1}^{0}<\rho_{1}$ and $0<\rho_{2}^{0}<\rho_{2}$.

(2) We denote by $\mathscr{H}$ the set of all the functions $w(t, x)$ satisfying the following properties:

i) $w(t, x)$ is a holomorphic function on $D_{r_{1}} \times D_{R_{1}}$ for some $0<r_{1}<r$, and $0<R_{1}<R$, and

ii) $|w(t, x)| \leq \rho_{1}^{0}$ and $|(\partial w / \partial x)(t, x)| \leq \rho_{2}^{0}$ hold on $D_{r_{1}} \times D_{R_{1}}$ for some $0<\rho_{1}^{0}<\rho_{1}$ and $0<\rho_{2}^{0}<\rho_{2}$.

Then we have

Theorem 4.4.2. $\quad$ Suppose the condition $\left(\mathrm{H}_{1}\right)$. The following two equations are equivalent:

$$
\begin{aligned}
& \frac{\partial u}{\partial t}=F\left(t, x, u, \frac{\partial u}{\partial x}\right) \quad \text { in } \mathscr{X}(\text { resp. in } \mathscr{H}), \\
& \frac{\partial w}{\partial t}=0 \quad \text { in } \mathscr{X}(\text { resp. in } \mathscr{H}) .
\end{aligned}
$$


This follows from Theorem 3.2.1 and

Proposition 4.4.3. (1) Let $\phi\left(t, x, u_{0}, u_{1}, \ldots\right)$ be the solution of $(\Phi)$ in (4.1.1). If $u \in \mathscr{X}$ (resp. $u \in \mathscr{H})$, then we have $\phi(t, x, u, \partial u / \partial x, \ldots) \in \mathscr{X}$ $($ resp. $\phi(t, x, u, \partial u / \partial x, \ldots) \in \mathscr{H})$.

(2) Let $\psi\left(t, x, w_{0}, w_{1}, \ldots\right)$ be the solution of $(\Psi)$ in (4.1.4). If $w \in \mathscr{X}$ (resp. $w \in \mathscr{H})$, then we have $\psi(t, x, w, \partial w / \partial x, \ldots) \in \mathscr{X}(\operatorname{resp} . \psi(t, x, w$, $\partial w / \partial x, \ldots) \in \mathscr{H})$.

Let us show this proposition now. For a function $w(t, x)=\sum_{l \geq 0} w_{l}(t) x^{l}$ we write $|w|_{x}(t, x)=\sum_{l \geq 0}\left|w_{l}(t)\right| x^{l}$. If $w(t, x) \in \mathscr{X}($ resp. $w(t, x) \in \mathscr{H})$ holds, the coefficients $w_{l}(t)$ are all holomorphic on $S_{\theta}\left(r_{1}\right)$ (resp. on $\left.D_{r_{1}}\right)$; but $\left|w_{l}(t)\right|$ $(l \geq 0)$ are only continuous on $S_{\theta}\left(r_{1}\right)$ (resp. on $D_{r_{1}}$ ). In order to treat this function $|w|_{x}(t, x)$, let us introduce the following function spaces $C(\mathscr{X})$ and $C(\mathscr{H})$ :

Definition 4.4.4. (1) We denote by $C(\mathscr{X})$ the set of all the functions $w(t, x)$ satisfying the following properties:

i) $w(t, x)$ is a continuous function on $S_{\theta}\left(r_{1}\right) \times D_{R_{1}}$ for some $\theta>0$, $0<r_{1}<r$ and $0<R_{1}<R$,

ii) $w(t, x)$ is holomorphic in $x \in D_{R_{1}}$ for any fixed $t \in S_{\theta}\left(r_{1}\right)$, and

iii) $|w(t, x)| \leq \rho_{1}^{0}$ and $|(\partial w / \partial x)(t, x)| \leq \rho_{2}^{0}$ hold on $S_{\theta}\left(r_{1}\right) \times D_{R_{1}}$ for some $0<\rho_{1}^{0}<\rho_{1}$ and $0<\rho_{2}^{0}<\rho_{2}$.

(2) We denote by $C(\mathscr{H})$ the set of all the functions $w(t, x)$ satisfying the following properties:

i) $w(t, x)$ is a continuous function on $D_{r_{1}} \times D_{R_{1}}$ for some $0<r_{1}<r$, and $0<R_{1}<R$

ii) $w(t, x)$ is holomorphic in $x \in D_{R_{1}}$ for any fixed $t \in D_{r_{1}}$, and

iii) $|w(t, x)| \leq \rho_{1}^{0}$ and $|(\partial w / \partial x)(t, x)| \leq \rho_{2}^{0}$ hold on $D_{r_{1}} \times D_{R_{1}}$ for some $0<\rho_{1}^{0}<\rho_{1}$ and $0<\rho_{2}^{0}<\rho_{2}$.

Then we have

Lemma 4.4.5. If $w(t, x) \in \mathscr{X}($ resp. $w(t, x) \in \mathscr{H})$ we have $|w|_{x}(t, x) \in$ $C(\mathscr{X})\left(\operatorname{resp} .|w|_{x}(t, x) \in C(\mathscr{H})\right)$.

Proof. Suppose that $w(t, x)=\sum_{l>0} w_{l}(t) x^{l} \in \mathscr{X}$. Then, by the conditions $|w(t, x)| \leq \rho_{1}^{0}$ and $|(\partial w / \partial x)(t, x)| \leq \rho_{2}^{0}$ on $S_{\theta}\left(r_{1}\right) \times D_{R_{1}}$ with $0<\rho_{1}^{0}<\rho_{1}$ and $0<\rho_{2}^{0}<\rho_{2}$ we have

$$
\left|w_{l}(t)\right| \leq \frac{\rho_{1}^{0}}{R_{1}{ }^{l}} \quad(l \geq 0) \quad \text { and } \quad\left|l w_{l}(t)\right| \leq \frac{\rho_{2}^{0}}{R_{1}{ }^{l-1}} \quad(l \geq 1),
$$


and so we see:

$$
\begin{aligned}
& |w|_{x}(t, x)=\sum_{l \geq 0}\left|w_{l}(t)\right| x^{l} \ll \sum_{l \geq 0} \frac{\rho_{1}^{0}}{R_{1}{ }^{l}} x^{l}=\frac{\rho_{1}^{0}}{1-x / R_{1}}, \\
& \frac{\partial|w|_{x}}{\partial x}(t, x)=\sum_{l \geq 1} l\left|w_{l}(t)\right| x^{l-1} \ll \sum_{l \geq 1} \frac{\rho_{2}^{0}}{R_{1}{ }^{l-1}} x^{l-1}=\frac{\rho_{2}^{0}}{1-x / R_{1}} .
\end{aligned}
$$

Therefore, if we take $R_{2}>0$ sufficiently small so that $\rho_{1}^{0} /\left(1-R_{2} / R_{1}\right)<\rho_{1}$ and $\rho_{2}^{0} /\left(1-R_{2} / R_{1}\right)<\rho_{2}$ we have the properties: i) $|w|_{x}(t, x)$ is a continuous function on $S_{\theta}\left(r_{1}\right) \times D_{R_{2}}$, ii) $|w|_{x}(t, x)$ is holomorphic in $x \in D_{R_{2}}$, and iii) $\left.|| w\right|_{x}(t, x) \mid \leq \rho_{1}^{*}$ and $\left|\left(\partial|w|_{x} / \partial x\right)(t, x)\right| \leq \rho_{2}^{*}$ on $S_{\theta}\left(r_{1}\right) \times D_{R_{2}}$ for $\rho_{1}^{*}=\rho_{1}^{0} /(1-$ $\left.R_{2} / R_{1}\right)<\rho_{1}$ and $\rho_{2}^{*}=\rho_{2}^{0} /\left(1-R_{2} / R_{1}\right)<\rho_{2}$. This proves the result: $|w|_{x}(t, x) \in$ $C(\mathscr{X})$. The case $\mathscr{H}$ can be proved in the same way.

Let

$$
\psi^{+}=w_{0}+\sum_{k \geq 1} \psi_{k}^{+}\left(x, w_{0}, \ldots, w_{k}\right) t^{k}
$$

be the unique solution of

$$
\frac{\partial \psi^{+}}{\partial t}=|F|\left(t, x, \psi^{+}, D\left[\psi^{+}\right]\right)
$$

If $w=w(t, x) \in \mathscr{X}$ (resp. $w=w(t, x) \in \mathscr{H})$, we have $|w|_{x}=|w|_{x}(t, x) \in$ $C(\mathscr{X})\left(\right.$ resp. $\left.|w|_{x}=|w|_{x}(t, x) \in C(\mathscr{H})\right)$ and

$$
\begin{aligned}
\phi_{k}(x, w, \partial w / \partial x, \ldots) & \ll\left|\phi_{k}\right|\left(x,|w|_{x}, \partial|w|_{x} / \partial x, \ldots\right) \\
& \ll \frac{k^{k}}{k !} \psi_{k}^{+}\left(x,|w|_{x}, \partial|w|_{x} / \partial x, \ldots\right), \quad k=1,2, \ldots \\
\psi_{k}(x, w, \partial w / \partial x, \ldots) & \ll\left|\psi_{k}\right|\left(x,|w|_{x}, \partial|w|_{x} / \partial x, \ldots\right) \\
& \ll \psi_{k}^{+}\left(x,|w|_{x}, \partial|w|_{x} / \partial x, \ldots\right), \quad k=1,2, \ldots
\end{aligned}
$$

as formal power series in $x$ (with a parameter $t$ ). Therefore, to prove Proposition 4.4.3 it is sufficient to show the following result.

Lemma 4.4.6. If $w=w(t, x) \in C(\mathscr{X})($ resp. $w=w(t, x) \in C(\mathscr{H})) w e$ have $\psi^{+}(t, x, w, \partial w / \partial x, \ldots) \in C(\mathscr{X})\left(\right.$ resp. $\left.\psi^{+}(t, x, w, \partial w / \partial x, \ldots) \in C(\mathscr{H})\right)$.

Proof. Let $\theta>0,0<r_{1}<r, 0<R_{1}<R, 0<\rho_{1}^{0}<\rho_{1}$ and $0<\rho_{2}^{0}<\rho_{2}$. Let $w(t, x)$ be a continuous function on $S_{\theta}\left(r_{1}\right) \times D_{R_{1}}$ which is holomorphic in $x \in$ 
$D_{R_{1}}$ for any fixed $t \in S_{\theta}\left(r_{1}\right)$ and satisfies $|w(t, x)| \leq \rho_{1}^{0}$ and $|(\partial w / \partial x)(t, x)| \leq \rho_{2}^{0}$ on $S_{\theta}\left(r_{1}\right) \times D_{R_{1}}$.

Then, by Proposition 4.2.5 we see that for any fixed $t \in S_{\theta}\left(r_{1}\right)$ and any fixed $s$ with $0<s<R_{1}$ the series

$$
\sum_{k \geq 1}\left\|\psi_{k}^{+}\left(x, w, \frac{\partial w}{\partial x}, \ldots, \frac{\partial^{k} w}{\partial x^{k}}\right)\right\|_{s}|z|^{k}
$$

is convergent in the domain

$$
\Omega_{s}^{+}=\left\{z \in \mathbb{C} ; \frac{|z|}{R_{1}-s}<\frac{r}{R_{1}+4 M^{+} r\left(R_{1} /\left(\rho_{1}-\rho_{1}^{0}\right)+e /\left(\rho_{2}-\rho_{2}^{0}\right)\right)}\right\} ;
$$

moreover we have the following estimates:

$$
\begin{aligned}
& \sum_{k \geq 1}\left\|\psi_{k}^{+}\left(x, w, \frac{\partial w}{\partial x}, \ldots, \frac{\partial^{k} w}{\partial x^{k}}\right)\right\|_{s}|z|^{k} \leq \frac{\rho_{1}-\rho_{1}^{0}}{2} \quad \text { on } \Omega_{s}^{+}, \\
& \sum_{k \geq 1}\left\|D\left[\psi_{k}^{+}\right]\left(x, w, \frac{\partial w}{\partial x}, \ldots, \frac{\partial^{k+1} w}{\partial x^{k+1}}\right)\right\|_{s}|z|^{k} \leq \frac{\rho_{2}-\rho_{2}^{0}}{2} \quad \text { on } \Omega_{s}^{+} .
\end{aligned}
$$

Thus, by setting $z=t$ we have

$$
\begin{aligned}
& \left|\psi^{+}\left(t, x, w, \frac{\partial w}{\partial x}, \ldots\right)\right| \\
& \quad \leq|w(t, x)|+\sum_{k \geq 1}\left\|\psi_{k}^{+}\left(x, w, \frac{\partial w}{\partial x}, \ldots, \frac{\partial^{k} w}{\partial x^{k}}\right)\right\|_{s}|t|^{k} \\
& \quad \leq \rho_{1}^{0}+\left(\rho_{1}-\rho_{1}^{0}\right) / 2<\rho_{1} \quad \text { on }\left(S_{\theta}\left(r_{1}\right) \cap \Omega_{s}^{+}\right) \times D_{s},
\end{aligned}
$$

and

$$
\begin{aligned}
& \left|\frac{\partial}{\partial x} \psi^{+}\left(t, x, w, \frac{\partial w}{\partial x}, \ldots\right)\right|=\left|D\left[\psi^{+}\right]\left(t, x, w, \frac{\partial w}{\partial x}, \ldots\right)\right| \\
& \leq\left|\frac{\partial w}{\partial x}(t, x)\right|+\sum_{k \geq 1}\left\|D\left[\psi_{k}^{+}\right]\left(x, w, \frac{\partial w}{\partial x}, \ldots, \frac{\partial^{k} w}{\partial x^{k}}\right)\right\|_{s}|t|^{k} \\
& \leq \rho_{2}^{0}+\left(\rho_{2}-\rho_{2}^{0}\right) / 2<\rho_{2} \quad \text { on }\left(S_{\theta}\left(r_{1}\right) \cap \Omega_{s}^{+}\right) \times D_{s} .
\end{aligned}
$$

This proves that $\psi^{+}(t, x, w, \partial w / \partial x, \ldots)$ belongs in the class $C(\mathscr{X})$.

Thus, the case $w \in C(\mathscr{X})$ is proved. The case $w \in C(\mathscr{H})$ can be proved in the same way.

Let us give an application. Suppose the condition $\left(\mathrm{H}_{1}\right)$. Let $u(t, x) \in \mathscr{X}$ be a solution of the equation

$$
\frac{\partial u}{\partial t}=F\left(t, x, u, \frac{\partial u}{\partial x}\right)
$$


Set $w(t, x)=\phi(t, x, u, \partial u / \partial x, \ldots)$. Then, $w(t, x) \in \mathscr{X}$ is a solution of

$$
\frac{\partial w}{\partial t}=0
$$

and so $w(t, x)$ can be expressed in the form $w(t, x)=h(x)$ for some holomorphic function $h(x)$ in a neighborhood of $x=0$. Since $w(t, x)=h(x) \in \mathscr{H}$ holds, by the reversibility we have $u(t, x)=\psi(t, x, w, \partial w / \partial x, \ldots)=\psi(t, x, h, \partial h / \partial x, \ldots)$ $\in \mathscr{H}$. Thus, we have $u(t, x) \in \mathscr{H}$. This proves

Theorem 4.4.7. $\quad$ Suppose the condition $\left(\mathrm{H}_{1}\right)$. If $u(t, x)$ is a solution of (4.4.6) belonging in the class $\mathscr{X}$, then $u(t, x)$ can be continued holomorphically up to some neighborhood of the origin of $\mathbb{C}_{t} \times \mathbb{C}_{x}$.

\section{$\S 5 . \quad$ Application}

Lastly, let us give an application to the problem of analytic continuation of the solution. We will prove the same result as in Kobayashi [4] and Lope-Tahara [5], as an application of the theory of coupling equations in this paper.

Let $\omega$ be an open neighborhood of the origin of $\mathbb{C}_{t} \times \mathbb{C}_{x}$. In this section we suppose

$\left(\mathrm{H}_{2}\right) \quad F\left(t, x, z_{1}, z_{2}\right)$ is a holomorphic function on $\omega \times \mathbb{C}_{z_{1}} \times \mathbb{C}_{z_{2}}$

and consider the equation

$$
\frac{\partial u}{\partial t}=F\left(t, x, u, \frac{\partial u}{\partial x}\right)
$$

By the Taylor expansion in $\left(z_{1}, z_{2}\right)$ we can express $F\left(t, x, z_{1}, z_{2}\right)$ in the form

$$
F\left(t, x, z_{1}, z_{2}\right)=\sum_{(j, \alpha) \in \Delta} a_{j, \alpha}(t, x) z_{1}{ }^{j} z_{2}{ }^{\alpha},
$$

where $(j, \alpha) \in \mathbb{N} \times \mathbb{N}, \Delta$ is a subset of $\mathbb{N} \times \mathbb{N}$, and $a_{j, \alpha}(t, x)$ are holomorphic functions on $\omega$. Without loss of generality we may suppose that $a_{j, \alpha}(t, x) \not \equiv 0$ for any $(j, \alpha) \in \Delta$; then we can write $a_{j, \alpha}(t, x)=t^{k_{j, \alpha}} b_{j, \alpha}(t, x)$, where $k_{j, \alpha}$ is a non-negative integer and $b_{j, \alpha}(0, x) \not \equiv 0$. Using the above, the function $F\left(t, x, z_{1}, z_{2}\right)$ may now be written as

$$
F\left(t, x, z_{1}, z_{2}\right)=\sum_{(j, \alpha) \in \Delta} t^{k_{j, \alpha}} b_{j, \alpha}(t, x) z_{1}{ }^{j} z_{2}{ }^{\alpha} .
$$


Set $\Delta_{2}=\{(j, \alpha) \in \Delta ; j+\alpha \geq 2\}$. We remark that the equation (5.0.1) is linear if and only if $\Delta_{2}=\emptyset$; it is nonlinear otherwise. In the case $\Delta_{2} \neq \emptyset$ we define the index $\sigma$ by

$$
\sigma=\sup _{(j, \alpha) \in \Delta_{2}} \frac{-k_{j, \alpha}-1}{j+\alpha-1}
$$

which was introduced by Kobayashi [4]. Note that this $\sigma$ is a non-positive real number.

Lemma 5.0.1. Set $\mathcal{M}=\left\{(j, \alpha) \in \Delta_{2} ; \sigma=\left(-k_{j, \alpha}-1\right) /(j+\alpha-1)\right\}$. We have:

(1) If $(j, \alpha) \in \mathcal{M}$ we have $k_{j, \alpha}+1+\sigma(j+\alpha-1)=0$.

(2) If $(j, \alpha) \in \Delta \backslash \mathcal{M}$ we have $k_{j, \alpha}+1+\sigma(j+\alpha-1)>0$.

Proof. (1) is clear from the definition of $\sigma$. Let us show (2). Let $(j, \alpha) \in$ $\Delta \backslash \mathcal{M}$; if $j+\alpha \geq 2$, by the definition of $\sigma$ we have $\sigma>\left(-k_{j, \alpha}-1\right) /(j+\alpha-1)$ which is equivalent to $k_{j, \alpha}+1+\sigma(j+\alpha-1)>0$, if $j+\alpha=1$ we have $k_{j, \alpha}+1+\sigma(j+\alpha-1)=k_{j, \alpha}+1 \geq 1$, and if $j+\alpha=0$ we have $k_{j, \alpha}+1+\sigma(j+$ $\alpha-1)=k_{j, \alpha}+1-\sigma \geq k_{j, \alpha}+1 \geq 1$.

\section{§5.1. Further equivalence of two PDEs}

Let $\sigma$ be the index defined by (5.0.2), and let us define a function-class $\mathscr{S}_{\sigma}$ whose elements can be singular at $t=0$.

Definition 5.1.1. We denote by $\mathscr{S}_{\sigma}$ the set of all the functions $w(t, x)$ satisfying the following properties:

i) $w(t, x)$ is a holomorphic function on $S_{\theta}(r) \times D_{R}$ for some $\theta>0$, $r>0$ and $R>0$, and

ii) $\|w(t)\|_{R}=o\left(|t|^{\sigma}\right)\left(\right.$ as $t \longrightarrow 0$ in $\left.S_{\theta}(r)\right)$.

Then we have

Theorem 5.1.2. Suppose the conditions $\left(\mathrm{H}_{2}\right)$ and $\Delta_{2} \neq \emptyset$; let $\sigma$ be the one in (5.0.2). The following two equations are equivalent:

$$
\begin{aligned}
& \frac{\partial u}{\partial t}=F\left(t, x, u, \frac{\partial u}{\partial x}\right) \quad \text { in } \mathscr{S}_{\sigma}, \\
& \frac{\partial w}{\partial t}=0 \quad \text { in } \mathscr{S}_{\sigma} .
\end{aligned}
$$


This follows from Theorem 3.2.1 and

Proposition 5.1.3. Suppose the conditions $\left(\mathrm{H}_{2}\right)$ and $\Delta_{2} \neq \emptyset$; let $\sigma$ be the one in (5.0.2). We have:

(1) Let $\phi\left(t, x, u_{0}, u_{1}, \ldots\right)$ be the solution of $(\Phi)$ in (4.1.1). If $u \in \mathscr{S}_{\sigma}$, then we have $\phi(t, x, u, \partial u / \partial x, \ldots) \in \mathscr{S}_{\sigma}$.

(2) Let $\psi\left(t, x, w_{0}, w_{1}, \ldots\right)$ be the solution of $(\Psi)$ in (4.1.4). If $w \in \mathscr{S}_{\sigma}$, then we have $\psi(t, x, w, \partial w / \partial x, \ldots) \in \mathscr{S}_{\sigma}$.

Proof. We will prove this by using Propositions 4.2.6 and 4.3.3. Since the proofs of (1) and (2) are quite parallel to each other, we will prove only the part (1).

Let $u(t, x)$ be a holomorphic function on $S_{\theta}\left(r_{0}\right) \times D_{R_{0}}$ with $\theta>0, r_{0}>0$ and $R_{0}>0$, and suppose that $\|u(t)\|_{R_{0}}=o\left(|t|^{\sigma}\right.$ ) (as $t \longrightarrow 0$ in $S_{\theta}$ ). We may assume that $r_{0}>0$ and $R_{0}>0$ are sufficiently small, and so we may also assume that $|F|\left(t, x, z_{1}, z_{2}\right)$ is holomorphic in a neighborhood of $\left\{\left(t, x, z_{1}, z_{2}\right) \in\right.$ $\mathbb{C}^{4} ;|t| \leq r_{0},|x| \leq R_{0},\left|z_{1}\right| \leq \rho_{1}$ and $\left.\left|z_{2}\right| \leq \rho_{2}\right\}$ for any $\rho_{1}>0$ and $\rho_{2}>0$. Hence, we may assume that

$$
\sum_{(j, \alpha) \in \Delta} r_{0}^{k_{j, \alpha}} B_{j, \alpha} \rho_{1}^{j} \rho_{2}{ }^{\alpha}<\infty
$$

holds for any $\rho_{1}>0$ and $\rho_{2}>0$, where

$$
B_{j, \alpha}=\max _{|t| \leq r_{0},|x| \leq R_{0}}|| b_{j, \alpha}|(t, x)|, \quad(j, \alpha) \in \Delta .
$$

Since $\|u(t)\|_{R_{0}}=o\left(|t|^{\sigma}\right)$ (as $t \longrightarrow 0$ in $S_{\theta}$ ) is assumed, we can choose a function $\epsilon_{t}$ (in $t \in S_{\theta}\left(r_{0}\right)$ ) such that $\|u(t)\|_{R_{0}} \leq \epsilon_{t}|t|^{\sigma}$ on $S_{\theta}\left(r_{0}\right)$ and that $\epsilon_{t}=o(1)$ (as $t \longrightarrow 0$ in $S_{\theta}$ ). Without loss of generality we may assume that $|t| / \epsilon_{t}=o(1)$ (as $t \longrightarrow 0$ in $S_{\theta}(r)$ ); if otherwise we have only to replace $\epsilon_{t}$ by $\max \left\{\epsilon_{t},|t|^{1 / 2}\right\}$.

Take any $0<R \leq R_{0} / 2$ and fix it. Then, take any $t \in S_{\theta}\left(r_{0} /(4 e)\right)$ and fix it. Set $u_{t}(x)=u(t, x)$; then $u_{t}(x)$ is a holomorphic function on $D_{R}$ and satisfies

$$
\left\|\frac{\partial^{k} u_{t}}{\partial x^{k}}\right\|_{R} \leq \frac{k ! \epsilon_{t}|t|^{\sigma}}{R^{k}}, \quad k=0,1,2, \ldots:
$$

this is verified by the conditions $R_{0}-R \geq R,\left\|u_{t}\right\|_{R_{0}} \leq \epsilon_{t}|t|^{\sigma}$ and Cauchy's inequality. 
Therefore, if we set

$$
\begin{aligned}
& r=4 e|t|, \quad \rho_{1}^{0}=2 \epsilon_{t}|t|^{\sigma}, \quad \rho_{1}=3 \epsilon_{t}|t|^{\sigma}, \quad \rho_{2}^{0}=4 \frac{\epsilon_{t}|t|^{\sigma}}{R}, \quad \rho_{2}=5 \frac{\epsilon_{t}|t|^{\sigma}}{R} \\
& \text { and } R_{1}=R, \quad \eta=R, \quad \varepsilon=\epsilon_{t}|t|^{\sigma}
\end{aligned}
$$

(where $t \in S_{\theta}\left(r_{0} /(4 e)\right)$ is fixed), we can apply Proposition 4.3.3 to this case and obtain the following result: the series

$$
\sum_{k \geq 1}\left\|\phi_{k}\right\|_{U_{k}} z^{k} \quad \text { with } U_{k}=U_{k}(R, \varepsilon, \eta)
$$

is convergent in the domain

$$
\Omega^{*}=\left\{z \in \mathbb{C} ;|z|<\frac{r}{e\left(3+4 M^{+} r\left((3+2 e) /\left(\epsilon_{t}|t|^{\sigma}\right)\right)\right.}\right\},
$$

where $M^{+}$is a constant satisfying

$$
|| F\left|\left(t, x, z_{1}, z_{2}\right)\right| \leq M^{+} \text {on } K^{*}
$$

and $K^{*}$ is the compact set $K^{*}=\left\{\left(t, x, z_{1}, z_{2}\right) \in \mathbb{C}^{4} ;|t| \leq r,|x| \leq R_{0},\left|z_{1}\right| \leq\right.$ $\left.\rho_{1},\left|z_{2}\right| \leq \rho_{2}\right\}$ under the condition (5.1.6). Note that the domain $\Omega^{*}$ depends on $t$. Since (5.1.5) is written as

$$
\left\|\frac{\partial^{k} u_{t}}{\partial x^{k}}\right\|_{R} \leq \frac{k ! \varepsilon}{\eta^{k}}, \quad k=0,1,2, \ldots \quad(\operatorname{under}(5.1 .6))
$$

we have

$$
\sum_{k \geq 1}\left\|\phi_{k}\left(x, u_{t}, \frac{\partial u_{t}}{\partial x}, \ldots, \frac{\partial^{k} u_{t}}{\partial x^{k}}\right)\right\|_{R}|z|^{k} \leq \sum_{k \geq 1}\left\|\phi_{k}\right\|_{U_{k}}|z|^{k}
$$

and so we see that the series of the left hand side is also convergent on $\Omega^{*}$.

If the fixed $t$ satisfies $t \in \Omega^{*}$, we can set $z=t$ and obtain the convergence of

$$
\sum_{k \geq 1} \phi_{k}\left(x, u_{t}, \frac{\partial u_{t}}{\partial x}, \ldots, \frac{\partial^{k} u_{t}}{\partial x^{k}}\right) t^{k}
$$

on $D_{R}$ (where $t$ is fixed).

Thus, to prove the convergence of $\phi(t, x, u(t, x),(\partial u / \partial x)(t, x), \ldots)$ on the domain $S_{\theta}(\mu) \times D_{R}$ it is sufficient to show the following lemma:

Lemma 5.1.4. There is a $\mu$ such that $0<\mu \leq r_{0} /(4 e)$ and that $t \in \Omega^{*}$ holds for any $t \in S_{\theta}(\mu)$; in other words, the inequality

$$
|t|<\frac{r}{e\left(3+4 M^{+} r\left((3+2 e) /\left(\epsilon_{t}|t|^{\sigma}\right)\right)\right.}
$$


holds for any $t \in S_{\theta}(\mu)$, where $r$ is the one in (5.1.6) and $M^{+}$is a constant satisfying (5.1.8).

Proof of Lemma 5.1.4. Since $r=4 e|t|$ we have only to show the inequality

$$
|t|<\frac{(4 e|t|)}{e\left(3+4 M^{+}(4 e|t|)\left((3+2 e) /\left(\epsilon_{t}|t|^{\sigma}\right)\right)\right.}
$$

which is equivalent to

$$
\frac{M^{+}|t|}{\epsilon_{t}|t|^{\sigma}}<\frac{1}{16 e(3+2 e)} .
$$

Since $|F|\left(t, x, z_{1}, z_{2}\right)$ is expressed in the form

$$
|F|\left(t, x, z_{1}, z_{2}\right)=\sum_{(j, \alpha) \in \Delta} t^{k_{j, \alpha}}\left|b_{j, \alpha}\right|(t, x) z_{1}^{j} z_{2}{ }^{\alpha}
$$

and since $K^{*}$ is the compact set $K^{*}=\left\{\left(t, x, z_{1}, z_{2}\right) \in \mathbb{C}^{4} ;|t| \leq r,|x| \leq\right.$ $\left.R_{0},\left|z_{1}\right| \leq \rho_{1},\left|z_{2}\right| \leq \rho_{2}\right\}$ we may take $M^{+}$as

$$
M^{+}=\sum_{(j, \alpha) \in \Delta} r^{k_{j, \alpha}} B_{j, \alpha} \rho_{1}^{j} \rho_{2}^{\alpha} \quad(\text { under }(5.1 .6))
$$

where $B_{j, \alpha}((j, \alpha) \in \Delta)$ are the constants in (5.1.4).

Let us show that the inequality (5.1.9) (with (5.1.10)) holds if $|t|$ is sufficiently small. By using $r=4 e|t|, \rho_{1}=3 \epsilon_{t}|t|^{\sigma}, \rho_{2}=5 \epsilon_{t}|t|^{\sigma} / R$ and by setting $A=\left(r_{0} / 4 e\right)^{1-\sigma}, c_{1}=\left(3 r_{0}{ }^{\sigma}\right) /(4 e)^{\sigma}, c_{2}=\left(5 r_{0}{ }^{\sigma}\right) /\left(R(4 e)^{\sigma}\right)$, we have

$$
\begin{aligned}
\frac{M^{+}|t|}{\epsilon_{t}|t|^{\sigma}} & =\sum_{(j, \alpha) \in \Delta}(4 e|t|)^{k_{j, \alpha}} B_{j, \alpha} 3^{j}(5 / R)^{\alpha}\left(\epsilon_{t}|t|^{\sigma}\right)^{j+\alpha-1}|t| \\
& =A \sum_{(j, \alpha) \in \Delta}\left(\frac{4 e}{r_{0}}|t|\right)^{k_{j, \alpha}+1+\sigma(j+\alpha-1)} \epsilon_{t}^{j+\alpha-1} r_{0}{ }^{k_{j, \alpha}} B_{j, \alpha} c_{1}{ }^{j} c_{2}{ }^{\alpha} \\
& =\sum_{(j, \alpha) \in \mathcal{M}} *+\sum_{\substack{(j, \alpha) \in \Delta \backslash \mathcal{M} \\
j+\alpha \geq 1}} *+\sum_{(j, \alpha)=(0,0)} * \\
& =I_{1}+I_{2}+I_{3} .
\end{aligned}
$$

In the case $(j, \alpha) \in \mathcal{M}$, we have $j+\alpha \geq 2$ and $k_{j, \alpha}+1+\sigma(j+\alpha-1)=0$. Since $|t|$ is sufficiently small we may assume that $\epsilon_{t} \leq 1$ holds. Therefore, we have

$$
\begin{aligned}
I_{1} & =A \sum_{(j, \alpha) \in \mathcal{M}} \epsilon_{t}{ }^{j+\alpha-1} r_{0}{ }^{k_{j, \alpha}} B_{j, \alpha} c_{1}{ }^{j} c_{2}{ }^{\alpha} \\
& \leq \epsilon_{t} A \sum_{(j, \alpha) \in \mathcal{M}} r_{0}{ }^{k_{j, \alpha}} B_{j, \alpha} c_{1}^{j} c_{2}{ }^{\alpha}=o(1) \quad(\text { as }|t| \longrightarrow 0)
\end{aligned}
$$


Since $t \in S_{\theta}\left(r_{0} /(4 e)\right)$ we have $\left(4 e / r_{0}\right)|t| \leq 1$. Therefore, by using $\epsilon_{t} \leq 1$ we have

$$
\begin{gathered}
I_{2} \leq A \sum_{\substack{(j, \alpha) \in \Delta \backslash \mathcal{M} \\
j+\alpha \geq 1}}\left(\frac{4 e}{r_{0}}|t|\right)^{k_{j, \alpha}+1+\sigma(j+\alpha-1)} r_{0}{ }^{k_{j, \alpha}} B_{j, \alpha} c_{1}{ }^{j} c_{2}{ }^{\alpha} \\
\leq A \sum_{\substack{(j, \alpha) \in \Delta \backslash \mathcal{M} \\
1 \leq j+\alpha \leq N}}\left(\frac{4 e}{r_{0}}|t|\right)^{k_{j, \alpha}+1+\sigma(j+\alpha-1)} r_{0}{ }^{k_{j, \alpha}} B_{j, \alpha} c_{1}{ }^{j} c_{2}{ }^{\alpha} \\
+A \sum_{j+\alpha>N} r_{0}{ }^{k_{j, \alpha}} B_{j, \alpha} c_{1}{ }^{j} c_{2}{ }^{\alpha}
\end{gathered}
$$

for any integer $N>0$. Since the second term of the right hand side is convergent, by choosing $N$ sufficiently large we can make it as small as possible. If we fix $N$, the first term of the right hand side is only a finite sum; therefore, by using the condition $k_{j, \alpha}+1+\sigma(j+\alpha-1)>0$ and by letting $|t| \longrightarrow 0$ we can make it as small as possible. Thus, we obtain the result: $I_{2}=o(1)$ (as $|t| \longrightarrow 0)$.

In the case $j+\alpha=0$ we have $k_{j, \alpha}+1+\sigma(j+\alpha-1)=k_{j, \alpha}+1-\sigma \geq$ $k_{j, \alpha}+1 \geq 1$ and so

$$
\begin{aligned}
I_{3} & =A \sum_{(j, \alpha)=(0,0)}\left(\frac{4 e}{r_{0}}|t|\right)^{k_{j, \alpha}+1+\sigma(j+\alpha-1)} \epsilon_{t}^{j+\alpha-1} r_{0}{ }^{k_{j, \alpha}} B_{j, \alpha} c_{1}{ }^{j} c_{2}^{\alpha} \\
& \leq A\left(\frac{4 e}{r_{0}}|t|\right) \epsilon_{t}{ }^{-1} r_{0}{ }^{k_{0,0}} B_{0,0} \\
& =\frac{|t|}{\epsilon_{t}} A\left(\frac{4 e}{r_{0}}\right) r_{0}{ }^{k_{0,0}} B_{0,0}=o(1) \quad(\text { as }|t| \longrightarrow 0) .
\end{aligned}
$$

In the last equality, we used the condition: $|t| / \epsilon_{t}=o(1)($ as $|t| \longrightarrow 0)$.

Thus, we have proved that

$$
\frac{M^{+}|t|}{\epsilon_{t}|t|^{\sigma}}=o(1) \quad(\text { as }|t| \longrightarrow 0)
$$

which implies that there is a $\mu>0$ such that (5.1.9) holds for any $|t| \leq \mu$. This proves Lemma 5.1.4.

Completion of the proof of Proposition 5.1.3. By Lemma 5.1.4 we have seen that $\phi(t, x, u(t, x),(\partial u / \partial x)(t, x), \ldots)$ is convergent on the domain $S_{\theta}(\mu) \times$ $D_{R}$. Moreover, by the latter half of Proposition 4.3.3 we have

$$
\sum_{k \geq 1}\left|\phi_{k}\left(x, u_{t}, \frac{\partial u_{t}}{\partial x}, \ldots, \frac{\partial^{k} u_{t}}{\partial x^{k}}\right)\right||t|^{k} \leq \frac{\rho_{1}-\rho_{1}^{0}}{2} \quad \text { on } S_{\theta}(\mu) \times D_{R} .
$$


Since $\rho_{1}-\rho_{1}^{0}=\epsilon_{t}|t|^{\sigma}$ we have

$$
\begin{aligned}
& \|\phi(t, x, u(t, x),(\partial u / \partial x)(t, x), \ldots)\|_{R} \\
& \quad \leq\|u(t)\|_{R}+\sum_{k \geq 1}\left\|\phi_{k}\left(x, u_{t}, \frac{\partial u_{t}}{\partial x}, \ldots, \frac{\partial^{k} u_{t}}{\partial x^{k}}\right)\right\|_{R}|t|^{k} \\
& \quad \leq \epsilon_{t}|t|^{\sigma}+\frac{\epsilon_{t}|t|^{\sigma}}{2}=o\left(|t|^{\sigma}\right) \quad(\text { as }|t| \longrightarrow 0) .
\end{aligned}
$$

Thus, we have obtained the condition $\phi(t, x, u(t, x),(\partial u / \partial x)(t, x), \ldots) \in \mathscr{S}_{\sigma}$. This completes the proof of Proposition 5.1.3.

\section{$\S 5.2 . \quad$ In the case $\|u(t)\|=O\left(|t|^{\sigma}\right)$}

Let $\mathcal{M}$ be as in Lemma 5.0.1. In this section we suppose

$$
\mathcal{M}=\emptyset:
$$

this implies that $k_{j, \alpha}+1+\sigma(j+\alpha-1)>0$ holds for all $(j, \alpha) \in \Delta$. Let us define a function-class $\mathscr{Z}_{\sigma}$ by the following:

Definition 5.2.1. We denote by $\mathscr{Z}_{\sigma}$ the set of all the functions $w(t, x)$ satisfying the following properties:

i) $w(t, x)$ is a holomorphic function on $S_{\theta}(r) \times D_{R}$ for some $\theta>0$, $r>0$ and $R>0$, and

ii) $\|w(t)\|_{R}=O\left(|t|^{\sigma}\right)\left(\right.$ as $t \longrightarrow 0$ in $\left.S_{\theta}(r)\right)$.

Then we have the following result.

Theorem 5.2.2. $\quad$ Suppose the condition $\left(\mathrm{H}_{2}\right)$. Let $\sigma$ be the one in (5.0.2) and suppose the condition (5.2.1). The following two equations are equivalent:

$$
\begin{aligned}
& \frac{\partial u}{\partial t}=F\left(t, x, u, \frac{\partial u}{\partial x}\right) \quad \text { in } \mathscr{Z}_{\sigma}, \\
& \frac{\partial w}{\partial t}=0 \quad \text { in } \mathscr{Z}_{\sigma} .
\end{aligned}
$$

To prove this, it is sufficient to show the following result:

Proposition 5.2.3. (1) Let $\phi\left(t, x, u_{0}, u_{1}, \ldots\right)$ be the solution of $(\Phi)$ in (4.1.1). If $u \in \mathscr{Z}_{\sigma}$, we have $\phi(t, x, u, \partial u / \partial x, \ldots) \in \mathscr{Z}_{\sigma}$.

(2) Let $\psi\left(t, x, w_{0}, w_{1}, \ldots\right)$ be the solution of $(\Psi)$ in (4.1.4). If $w \in \mathscr{Z}_{\sigma}$, we have $\psi(t, x, w, \partial w / \partial x, \ldots) \in \mathscr{Z}_{\sigma}$. 
Proof. In this case, the proof is quite parallel to the proof of Proposition 5.1.3; we have only to notice that " $=o\left(|t|^{\sigma}\right) "$ is replaced by " $=O\left(|t|^{\sigma}\right) "$, and so we have only to replace $\epsilon_{t}$ by a positive constant $C>0$ in the proof of Proposition 5.1.3. Thus, by using the conditions $k_{j, \alpha}+1+\sigma(j+\alpha-1)>0$ (for $(j, \alpha) \in \Delta)$ and $\left(4 e / r_{0}\right)|t| \leq 1$ we can estimate $\left(M^{+}|t|\right) /\left(C|t|^{\sigma}\right)$ as follows:

$$
\begin{aligned}
& \frac{M^{+}|t|}{C|t|^{\sigma}}=\sum_{(j, \alpha) \in \Delta}(4 e|t|)^{k_{j, \alpha}} B_{j, \alpha} 3^{j}(5 / R)^{\alpha}\left(C|t|^{\sigma}\right)^{j+\alpha-1}|t| \\
& =A \sum_{(j, \alpha) \in \Delta}\left(\frac{4 e}{r_{0}}|t|\right)^{k_{j, \alpha}+1+\sigma(j+\alpha-1)} C^{j+\alpha-1} r_{0}{ }^{k_{j, \alpha}} B_{j, \alpha} c_{1}{ }^{j} c_{2}{ }^{\alpha} \\
& \leq A \sum_{\substack{(j, \alpha) \in \Delta \\
j+\alpha \leq N}}\left(\frac{4 e}{r_{0}}|t|\right)^{k_{j, \alpha}+1+\sigma(j+\alpha-1)} C^{j+\alpha-1} r_{0}{ }^{k_{j, \alpha}} B_{j, \alpha} c_{1}{ }^{j} c_{2}{ }^{\alpha} \\
& +A \sum_{\substack{(j, \alpha) \in \Delta \\
j+\alpha>N}} C^{j+\alpha-1} r_{0}{ }^{k_{j, \alpha}} B_{j, \alpha} c_{1}{ }^{j} c_{2}^{\alpha} \\
& =I_{1}+I_{2} \text {. }
\end{aligned}
$$

We note that the series $I_{2}$ is convergent; therefore, by taking $N$ sufficiently large we can make $I_{2}$ as small as we want; if $N$ is fixed, the term $I_{1}$ is a finite sum and so by using the condition $k_{j, \alpha}+1+\sigma(j+\alpha-1)>0$ we see: $I_{1}=o(1)$ (as $|t| \longrightarrow 0)$. Thus, if $|t|>0$ is sufficiently small we have

$$
\frac{M^{+}|t|}{C|t|^{\sigma}}<\frac{1}{16 e(3+2 e)} .
$$

This corresponds to the result in (5.1.9).

The other parts of the proof of Proposition 5.2.3 is the same as in the proof of Proposition 5.1.3, and so we may omit the details.

\section{§5.3. Analytic continuation}

Let us give an application. Let $u(t, x) \in \mathscr{S}_{\sigma}\left(\operatorname{resp} . \quad u(t, x) \in \mathscr{Z}_{\sigma}\right)$ be a solution of the equation

$$
\frac{\partial u}{\partial t}=F\left(t, x, u, \frac{\partial u}{\partial x}\right)
$$

Set $w(t, x)=\phi(t, x, u, \partial u / \partial x, \ldots)$. Then, $w(t, x) \in \mathscr{S}_{\sigma}\left(\operatorname{resp} . w(t, x) \in \mathscr{Z}_{\sigma}\right)$ is a solution of

$$
\frac{\partial w}{\partial t}=0
$$


and so $w(t, x)$ can be expressed in the form $w(t, x)=h(x)$ for some holomorphic function $h(x)$ in a neighborhood of $x=0$. Since $w(t, x)=h(x) \in \mathscr{H}$ holds, by Theorem 4.4 .2 we have $u(t, x)=\psi(t, x, h, \partial h / \partial x, \ldots) \in \mathscr{H}$. This proves

Theorem 5.3.1. $\quad$ Suppose the conditions $\left(\mathrm{H}_{2}\right)$ and $\Delta_{2} \neq \emptyset$; let $\sigma$ be the one in (5.0.2). We have:

(1) If $u(t, x)$ is a solution of (5.0.1) belonging in the class $\mathscr{S}_{\sigma}$, then $u(t, x)$ can be continued holomorphically up to some neighborhood of the origin of $\mathbb{C}_{t} \times$ $\mathbb{C}_{x}$.

(2) If $\mathcal{M}=\emptyset$ and if $u(t, x)$ is a solution of (5.0.1) belonging in the class $\mathscr{Z}_{\sigma}$, then $u(t, x)$ can be continued holomorphically up to some neighborhood of the origin of $\mathbb{C}_{t} \times \mathbb{C}_{x}$.

Note that this is just the result of the first order case in Kobayashi [4] and Lope-Tahara [5]. It is shown in Tahara [7] and [8] that the above result is optimal for the analytic continuation of the solution. We will give here only the following example.

Example 5.3.2. $\quad$ In the case

$$
\frac{\partial u}{\partial t}=u\left(\frac{\partial u}{\partial x}\right)^{m} \quad(\text { with } m \in\{1,2, \ldots\})
$$

we have $\sigma=-1 / m$ and $\mathcal{M} \neq \emptyset$. We see:

(1) If $u(t, x)$ is a solution belonging in the class $\mathscr{S}_{\sigma}$ (with $\left.\sigma=-1 / m\right)$, then $u(t, x)$ can be continued holomorphically up to some neighborhood of the origin of $\mathbb{C}_{t} \times \mathbb{C}_{x}$.

(2) But, we have a solution

$$
u(t, x)=\left(\frac{-1}{m}\right)^{1 / m} \frac{x+c}{t^{1 / m}} \quad(\text { where } c \in \mathbb{C} \text { is an arbitrary constant) }
$$

which belongs in the class $\mathscr{Z}_{\sigma}$ (with $\sigma=-1 / m$ ) and which cannot be continued holomorphically to any neighborhood of the origin. Since this solution has a singularity on $\{t=0\}$ of order $|t|^{-1 / m}$, this does not belong in the class $\mathscr{S}_{\sigma}$ (with $\sigma=-1 / m$ ).

\section{References}

[1] R. Gérard and H. Tahara, Singular nonlinear partial differential equations, Vieweg, Braunschweig, 1996. 
[2] E. Hille, Ordinary differential equations in the complex domain, Wiley-Intersci., New York, 1976.

[3] L. Hörmander, Linear partial differential operators, Springer, Berlin, 1976.

[4] T. Kobayashi, Singular solutions and prolongation of holomorphic solutions to nonlinear differential equations, Publ. Res. Inst. Math. Sci. 34 (1998), no. 1, 43-63.

[5] J. E. C. Lope and H. Tahara, On the analytic continuation of solutions to nonlinear partial differential equations, J. Math. Pures Appl. 81 (2002), no. 9, 811-826.

[6] M. Nagumo, Über das Anfangswertproblem partieller Differentialgleichungen, Jap. J. Math. 18 (1942), 41-47.

[7] H. Tahara, On the singularities of solutions of nonlinear partial differential equations in the complex domain, in Microlocal analysis and complex Fourier analysis, 273-283, World Sci. Publ., River Edge, NJ, 2002.

[8] _ On the singularities of solutions of nonlinear partial differential equations in the complex domain. II, in Differential equations 8 asymptotic theory in mathematical physics, 343-354, World Sci. Publ., Hackensack, NJ, 2004.

[9] Coupling of two partial differential equations and its application, to appear in Proceedings of the Conference on Algebraic Analysis of Differential Equations (held at Kyoto in July 07-14, 2005), Springer. 